\title{
Isoperimetric Inequalities for Non-Local Dirichlet Forms
}

\author{
Feng-Yu Wang ${ }^{a, b)}$ and Jian Wang ${ }^{c)}$ \\ ${ }^{a)}$ Center of Applied Mathematics, Tianjin University, Tianjin 300072, China \\ ${ }^{b)}$ Department of Mathematics, Swansea University, Singleton Park, SA2 8PP, United Kingdom \\ ${ }^{c}$ College of Mathematics and Informatics, Fujian Normal University, Fuzhou 350007, China \\ wangfy@tju.edu.cn, F.-Y.Wang@swansea.ac.uk, jianwang@fjnu.edu.cn
}

October 6, 2019

\begin{abstract}
Let $(E, \mathscr{F}, \mu)$ be a $\sigma$-finite measure space. For a non-negative symmetric measurable function $J(x, y)$ on $E \times E$, consider the quadratic form

$$
\mathscr{E}(f, f):=\frac{1}{2} \int_{E \times E}(f(x)-f(y))^{2} J(x, y) \mu(\mathrm{d} x) \mu(\mathrm{d} y)
$$

in $L^{2}(\mu)$. We characterize the relationship between the isoperimetric inequality and the super Poincaré inequality associated with $\mathscr{E}$. In particular, sharp Orlicz-Sobolev type and Poincaré type isoperimetric inequalities are derived for stable-like Dirichlet forms on $\mathbb{R}^{n}$, which include the existing fractional isoperimetric inequality as a special example.
\end{abstract}

AMS subject Classification: 47G20, 47D62.

Keywords: Isoperimetric inequality, non-local Dirichlet form, super Poincaré inequality, Orlicz norm.

\section{Introduction}

For local (i.e. differential) quadratic forms, the isoperimetric inequality is a geometric inequality using the surface area of a set to bound its volume, see, for instance $[21,35,37]$ and references therein, for the study of isoperimetric inequalities and applications to symmetric diffusion processes. In this case, the surface area refers to the possibility for the associated diffusion process to exit the set. 
In the non-local case, the associated process is a jump process which exits a set without hitting the boundary, so it is reasonable to replace the surface area of a set $A$ by the jump rate from $A$ to its complementary $A^{c}$. In this spirit, the famous Cheeger inequality [14] for the first eigenvalue was extended in [30, 17] to jump processes (see also [43] for finite Markov chains). See $[15,16,51,53,42,50,32,33]$ for the study of more general functional inequalities of symmetric jump processes using isoperimetric constants. These references only consider large jumps (i.e. the total jump rate is finite). In this paper, we aim to investigate isoperimetric inequalities for non-local forms with infinite jump rates, for which small jumps will play a key role.

To explain our motivation more clearly, let us start from the following classical isoperimetric inequality on $\mathbb{R}^{n}$ :

$$
\mu_{\partial}(\partial A) \geq n \mu(A)^{\frac{n-1}{n}} \omega_{n}^{\frac{1}{n}}
$$

where $A$ is a measurable subset of $\mathbb{R}^{n}$ with finite volume, $\partial A$ is its boundary, $\omega_{n}$ is the volume of the $n$-dimensional unit ball, $\mu$ is the Lebesgue measure and $\mu_{\partial}$ is the area measure induced by $\mu$ :

$$
\mu_{\partial}(\partial A):=\limsup _{\varepsilon \downarrow 0} \frac{\mu(\{\operatorname{dist}(\cdot, A) \leq \varepsilon\})-\mu(A)}{\varepsilon} .
$$

In particular, the equality in (1.1) holds for $A$ being a ball. By the co-area formula, (1.1) is equivalent to the sharp $L^{1}$-Sobolev inequality (i.e. the energy form is of $L^{1}$ type)

$$
\|f\|_{\frac{n}{n-1}} \leq \frac{1}{n \omega_{n}^{1 / n}} \int_{\mathbb{R}^{n}}|\nabla f(x)| \mathrm{d} x, \quad f \in W^{1,1}\left(\mathbb{R}^{n}\right),
$$

where for any $p \geq 1,\|f\|_{p}:=\left(\int_{\mathbb{R}^{n}}|f(x)|^{p} \mathrm{~d} x\right)^{1 / p}$ and $W^{1, p}\left(\mathbb{R}^{n}\right)$ is the homogeneous Sobolev space of differentiability 1 and integrability $p$. For $n>2$, applying (1.2) to $f=|g|^{\frac{2(n-1)}{n-2}}$ and using the Cauchy-Schwarz inequality, we obtain the sharp Sobolev inequality:

$$
\|g\|_{\frac{2 n}{n-2}} \leq \frac{2(n-1)}{n(n-2) \omega_{n}^{1 / n}}\left(\int_{\mathbb{R}^{n}}|\nabla g(x)|^{2} \mathrm{~d} x\right)^{1 / 2}, \quad g \in W^{1,2}\left(\mathbb{R}^{n}\right) .
$$

These inequalities are also available for the $\alpha$-stable Dirichlet form. For any $\alpha \in(0,2 \wedge n)$, there exists a universal constant $C>0$ such that the fractional Sobolev inequality

$$
\|f\|_{\frac{2 n}{n-\alpha}} \leq C\left(\int_{\mathbb{R}^{n} \times \mathbb{R}^{n}} \frac{(f(x)-f(y))^{2}}{|x-y|^{n+\alpha}} \mathrm{d} x \mathrm{~d} y\right)^{1 / 2}, \quad f \in C_{c}^{\infty}\left(\mathbb{R}^{n}\right)
$$

holds. By an approximation argument, this inequality can be extended to $f \in W^{\alpha / 2,2}\left(\mathbb{R}^{d}\right)$. Here and in what follows, for $p \geq 1, W^{\alpha / 2, p}\left(\mathbb{R}^{d}\right)$ is denoted by the fractional homogeneous Sobolev space, which is the completion of $C_{c}^{\infty}\left(\mathbb{R}^{n}\right)$ with respect to

$$
\left(\int_{\mathbb{R}^{n} \times \mathbb{R}^{n}} \frac{|f(x)-f(y)|^{p}}{|x-y|^{n+p \alpha / 2}} \mathrm{~d} x \mathrm{~d} y\right)^{1 / p} .
$$


Correspondingly to (1.2), [36, Theorem 1.1] (or [27, Theorem 4.1] with sharp constant) gives the following $L^{1}$-Sobolev inequality

$$
\|f\|_{\frac{n}{n-\alpha / 2}} \leq C \int_{\mathbb{R}^{n} \times \mathbb{R}^{n}} \frac{|f(x)-f(y)|}{|x-y|^{n+\alpha / 2}} \mathrm{~d} x \mathrm{~d} y, \quad f \in W^{\alpha / 2,1}\left(\mathbb{R}^{n}\right)
$$

for some constant $C>0$. The proof of (1.3) addressed in [36, 27] relies on the Hardy inequality for fractional Sobolev spaces. We note that the Sobolev embedding theorems involving the spaces $W_{\alpha / 2, p}$ also can be obtained by interpolation techniques and by passing through Besov spaces, see for example [7,8]. For the treatment of fractional Sobolev-type inequalities we can refer to $[1,5,46,40]$ and the references therein.

According to Theorem 2.1(1) below, (1.3) holds if and only if

$$
\kappa:=\inf _{\mu(A) \in(0, \infty)} \frac{1}{\mu(A)^{\frac{n-\alpha / 2}{n}}} \int_{A \times A^{c}} \frac{\mathrm{d} x \mathrm{~d} y}{|x-y|^{n+\alpha / 2}}>0,
$$

and furthermore, $\kappa \in\left[\frac{1}{2 C}, \frac{n}{2 C(n-\alpha / 2)}\right]$, where $C$ is the sharp constant in (1.3). Due to this fact, we also call (1.3) a Sobolev type isoperimetric inequality. In the literature (e.g. see $[2,10,12,13,49])$, for any $s \in(0,1)$ and Borel set $A \subset \mathbb{R}^{n}$,

$$
P_{s}(A):=\int_{A \times A^{c}} \frac{\mathrm{d} x \mathrm{~d} y}{|x-y|^{n+s}}
$$

is called the fractional $s$-perimeter of $A$. The limiting behaviors of fractional $s$-perimeters as $s \rightarrow 1-$ and as $s \rightarrow 0+$ have attracted a lot of attention. The reader can be referred to $[23,10]$ for the case $s \rightarrow 1-$, and $[36,24]$ for $s \rightarrow 0+$. The other important result for $s$-perimeters is the fractional isoperimetric inequality, which is closely related to the constant $\kappa$ defined by (1.4). An isoperimetric type inequality for $s$-perimeters has been proved by Frank and Seiringer in [27], and later the corresponding stability version was established in $[28,26]$.

In this paper, we aim to establish isoperimetric inequalities for the following non-local form on a $\sigma$-finite measure space $(E, \mathscr{F}, \mu)$ :

$$
\mathscr{E}(f, f):=\frac{1}{2} \int_{E \times E}(f(x)-f(y))^{2} J(x, y) \mu(\mathrm{d} x) \mu(\mathrm{d} y),
$$

where $J(x, y)$ is a non-negative symmetric measurable function on $E \times E$.

Instead of the fractional Hardy inequality used in $[36,27]$ and the Besov or interpolation spaces used in $[7,8]$, in our paper we will apply the super Poincaré inequality of $\mathscr{E}$, which was introduced by the first author in [51]. This inequality can be regarded as a deformation of the Nash-type inequality, but is easier to verify in applications. The proof here is self-contained.

We also mentioned that isoperimetric inequalities for symmetric diffusions have already been studied in the literature, see $[11,31,51,3,4]$ and the references therein. In particular, Ledoux's approach of Buser's inequality was used in $[51,3]$ to illustrate the relation 
of the super-Poincaré inequalities with isoperimetry. A notion of Orlicz hypercontractive semigroups was introduced in [3], and their relations with various functional inequalities were studied. A measure-Capacity sufficient condition, in the spirit of Maz'ja [35], was established for super-Poincaré inequality inequality in [4]. In the present setting, we are concerned with non-local forms. We will directly derive the equivalence of $L^{1}$ Orlicz-Sobolev inequality (involving the $L^{1}$-norm of the jumping kernel for non-local forms) and $L^{1}$-Poincaré type inequality, and also characterize the relationship between the isoperimetric inequality and the super Poincaré inequality. In particular, one of our general results (see Theorem 2.2 below) implies the following Orlicz-Sobolev type isoperimetric inequality (1.7) on $\mathbb{R}^{n}$.

Following [41, Section 1.3], a function $N:[0, \infty) \rightarrow[0, \infty]$ is called a Young function if it is convex and increasing with $N(0)=0$ and $N(\infty):=\lim _{s \rightarrow \infty} N(s)=\infty$. We consider the following Orlicz norm induced by $N$ (see [41, Section 3.2]):

$$
\|f\|_{N}:=\inf \left\{r>0: \int_{\mathbb{R}^{n}} N\left(\frac{|f(x)|}{r}\right) \mathrm{d} x \leq 1\right\}
$$

where $\inf \emptyset=\infty$ by convention. Let $L_{N}\left(\mathbb{R}^{n}\right)=\left\{f \in \mathscr{B}\left(\mathbb{R}^{n}\right):\|f\|_{N}<\infty\right\}$. It is easy to see from the convexity and $N(0)=0$ that $c N(s) \leq N(c s)$ for $c \geq 1$. So, $N(\infty)>0$ is equivalent to $N(\infty)=\infty$, and

$$
c^{-1}\|f\|_{c N} \leq\|f\|_{N} \leq\|f\|_{c N}, \quad c \geq 1
$$

For two Young functions $N_{1}$ and $N_{2}$, we say that $N_{1}$ is not dominated by $N_{2}$ if $\sup _{s>0} \frac{N_{1}(s)}{N_{2}(s)}=$ $\infty$, where we set $\frac{0}{0}=1, \frac{\infty}{\infty}=1, \frac{r}{0}=\infty$ and $\frac{r}{\infty}=0$ for $r>0$. In this case, we write $N_{1} \npreceq N_{2}$.

For $\alpha \in(0,2)$, let $\mathscr{H}_{\alpha}$ be the class of functions $h:(0, \infty) \rightarrow(0, \infty)$ satisfying

(i) $h(s)$ and $\operatorname{sh}(s)^{-1}$ are increasing in $s$.

(ii) For any $s>0$,

$$
\Phi_{h}(s):=\int_{0}^{s} \mathrm{~d} t \int_{0}^{t^{-1 / n}} \frac{r^{\alpha-1}}{h(r)} \mathrm{d} r<\infty
$$

It is easy to see that $\Phi_{h}$ is continuous, strictly increasing and concave with $\Phi_{h}(0)=0$. Thus, $N_{h}:=\Phi_{h}^{-1}$ is a Young function.

Theorem 1.1. For any $\alpha \in(0,2)$ and $h \in \mathscr{H}_{\alpha}$, there exists a constant $C>0$ such that

$$
\|f\|_{N_{h}} \leq C \int_{\mathbb{R}^{n} \times \mathbb{R}^{n}}|f(x)-f(y)| \frac{h(|x-y|)}{|x-y|^{n+\alpha}} \mathrm{d} x \mathrm{~d} y, \quad f \in L_{N_{h}}\left(\mathbb{R}^{n}\right),
$$

which implies

$$
\inf _{\mu(A) \in(0, \infty)}\left(N_{h}^{-1}\left(\mu(A)^{-1}\right) \int_{A \times A^{c}} \frac{h(|x-y|)}{|x-y|^{n+\alpha}} \mathrm{d} x \mathrm{~d} y\right)>0
$$

Consequently: 
(1) For any $\alpha_{1}, \alpha_{2} \in(0,2)$, let

$$
N_{\alpha_{1}, \alpha_{2}}^{\wedge}(s):=s^{\frac{n}{n-\alpha_{1} / 2}} \wedge s^{\frac{n}{n-\alpha_{2} / 2}}, \quad N_{\alpha_{1}, \alpha_{2}}^{\vee}(s):=s^{\frac{n}{n-\alpha_{1} / 2}} \vee s^{\frac{n}{n-\alpha_{2} / 2}}, \quad s \geq 0 .
$$

Then there exists a constant $C>0$ such that

$$
\begin{aligned}
\|f\|_{N_{\alpha_{1}, \alpha_{2}}^{\wedge}} & \leq C \int_{\mathbb{R}^{n} \times \mathbb{R}^{n}} \frac{|f(x)-f(y)|}{|x-y|^{n+\alpha_{1} / 2} \vee|x-y|^{n+\alpha_{2} / 2}} \mathrm{~d} x \mathrm{~d} y, \quad f \in L_{N_{\alpha_{1}, \alpha_{2}}^{\wedge}}\left(\mathbb{R}^{n}\right), \\
\|f\|_{N_{\alpha_{1}, \alpha_{2}}^{\vee}} & \leq C \int_{\mathbb{R}^{n} \times \mathbb{R}^{n}} \frac{|f(x)-f(y)|}{|x-y|^{n+\alpha_{1} / 2} \wedge|x-y|^{n+\alpha_{2} / 2}} \mathrm{~d} x \mathrm{~d} y, \quad f \in L_{N_{\alpha_{1}, \alpha_{2}}^{\vee}}\left(\mathbb{R}^{n}\right) .
\end{aligned}
$$

These inequalities are sharp in the sense that (1.9) (resp. (1.10)) fails if $N_{\alpha_{1}, \alpha_{2}}^{\wedge}$ (resp. $N_{\alpha_{1}, \alpha_{2}}^{\vee}$ ) is replaced by a Young function $N \npreceq N_{\alpha_{1}, \alpha_{2}}^{\wedge}\left(\right.$ resp. $\left.N_{\alpha_{1}, \alpha_{2}}^{\vee}\right)$.

(2) For any $\alpha \in(0,2)$ and $q, p \in \mathbb{R}$, let $\lambda \geq 2$ large enough such that both $N_{\alpha}^{\text {log,q,+}}(s):=$ $\left\{s \log ^{q}(\lambda+s)\right\}^{\frac{n}{n-\alpha / 2}}$ and $N_{\alpha}^{\log , p,-}(s):=\left\{s \log ^{p}\left(\lambda+s^{-1}\right)\right\}^{\frac{n}{n-\alpha / 2}}$ are Young functions. Then there exists a constant $C>0$ such that for all $f \in L_{N_{\alpha}^{l o g, q,+}}\left(\mathbb{R}^{n}\right)$,

$$
\|f\|_{N_{\alpha}^{l o g, q,+}} \leq C \int_{\mathbb{R}^{n} \times \mathbb{R}^{n}}|f(x)-f(y)| \frac{\left\{\log \left(2+|x-y|^{-1}\right)\right\}^{q}}{|x-y|^{n+\alpha / 2}} \mathrm{~d} x \mathrm{~d} y
$$

and for all $f \in L_{N_{\alpha}^{l o g, p,-}}\left(\mathbb{R}^{n}\right)$,

$$
\|f\|_{N_{\alpha}^{l o g, p,-}} \leq C \int_{\mathbb{R}^{n} \times \mathbb{R}^{n}}|f(x)-f(y)| \frac{\{\log (2+|x-y|)\}^{p}}{|x-y|^{n+\alpha / 2}} \mathrm{~d} x \mathrm{~d} y
$$

These inequalities are sharp in the sense that (1.11) (resp. (1.12)) fails if $N_{\alpha}^{\text {log,q,+ }}$ (resp. $\left.N_{\alpha}^{\text {log,p,-}}\right)$ is replaced by a Young function $N \npreceq N_{\alpha}^{\text {log,q,+}}\left(\operatorname{resp} . N_{\alpha}^{\text {log,p,-}}\right)$.

According to Theorem 2.1(1) below, (1.7) and (1.8) are equivalent in more general case, so an $L^{1}$ Orlicz-Sobolev inequality of type (1.7) is also called an Orlicz-Sobolev type isoperimetric inequality. It is easy to see that when $\alpha_{1}=\alpha_{2}=\alpha$ and $q=p=0$, the inequalities (1.9), (1.10), (1.11) and (1.12) coincide with (1.3). The Orlicz-Sobolev type isoperimetric inequalities (1.9)-(1.12) are equivalent to the corresponding Poincaré type ones, see Corollary 2.7 for details.

The inequalities (1.9), (1.10), (1.11) and (1.12) are seen as variants of the $L^{1}$-Sobolev inequality (1.3) for standard $\alpha$-stable-like Dirichlet forms on $\mathbb{R}^{n}$. One of our contributions is to establish the $L^{1}$ Orlicz-Sobolev inequality (2.3) as well as the corresponding Poincaré type inequality for general non-local Drichlet forms. In particular, given $\alpha \in(0,2)$ and $h \in \mathscr{H}_{\alpha}$, we are mainly concerned on the Orlicz-Sobolev type isoperimetric inequality (1.7) on $\mathbb{R}^{d}$ with the qualitatively-optimal Young function $N_{h}$. However, our results are not robust in the sense that the corresponding results for local Dirichlet forms can not be covered from them by letting $\alpha \rightarrow 2-$, since the corresponding constants involved in these inequalities 
may tend to infinite as $\alpha \rightarrow 2-$. See Remark 4.6 for additional comments on non-local Dirichlet forms with finite reference measures.

In the remainder of the paper, we will work with the form (1.5) under a general framework. In Section 2, we characterize the link between the super poincaré and isoperimteric inequalities. In Section 3, we first apply the main result derived in Section 2 to prove Theorem 1.1, then make extensions to the truncated and discrete $\alpha$-stable Dirichlet forms. Finally, by using a perturbation argument, we derive isoperimetric inequalities in Section 4 for $\alpha$-stable-like Dirichlet forms with finite reference measures.

\section{Super Poincaré and isoperimetric inequalities: gen- eral results}

Let $(E, \mathscr{F}, \mu)$ be a $\sigma$-finite measure space, and let $J(x, y)$ be a non-negative symmetric measurable function on $E \times E$. In this section, we investigate the link between the isoperimetric inequality and the super Poincaré inequality for the following symmetric quadratic form

$$
\begin{aligned}
& \mathscr{E}(f, g):=\frac{1}{2} \int_{E \times E}(f(x)-f(y))(g(x)-g(y)) J(x, y) \mu(\mathrm{d} x) \mu(\mathrm{d} y), \\
& f, g \in \mathscr{D}(\mathscr{E}):=\left\{f \in L^{2}(\mu): \mathscr{E}(f, f)<\infty\right\} .
\end{aligned}
$$

Moreover, we assume that $\mathscr{D}(\mathscr{E})$ is dense in $L^{2}(\mu)$ so that $(\mathscr{E}, \mathscr{D}(\mathscr{E}))$ is a symmetric Dirichlet form.

According to [51], we say that $(\mathscr{E}, \mathscr{D}(\mathscr{E}))$ satisfies the super Poincaré inequality with rate function $\beta:(0, \infty) \rightarrow(0, \infty)$, if

$$
\|f\|_{2}^{2} \leq r \mathscr{E}(f, f)+\beta(r)\|f\|_{1}^{2}, \quad f \in \mathscr{D}(\mathscr{E}), r>0 .
$$

Here and in what follows, for any $p \in[1, \infty],\|\cdot\|_{p}$ denotes the $L^{p}$-norm with respect to $\mu$. Since $\mathscr{E}(f, f) \geq 0$, we may and do assume that $\beta$ is decreasing on $(0, \infty)$. See $[51,52,54]$ and references within for the super Poincaré inequality and applications.

For a Young function $N$, let $\|\cdot\|_{N}$ be the Orlicz norm induced by $N$ and the measure $\mu$, and let $L_{N}(\mu)=\left\{f \in \mathscr{B}(E):\|f\|_{N}<\infty\right\}$, where $\mathscr{B}(E)$ is the class of measurable functions on $E$. The left derivative of Young function $N$, denoted by $N_{-}^{\prime}$, always exists and is non-decreasing left continuous on $(0, \infty)$, see e.g. [41, Section 1.3]. For any non-negative decreasing function $f$ on $[0, \infty)$, let

$$
f^{-1}(r):=\inf \{s>0: f(s) \leq r\}, \quad r \geq 0,
$$

where $\inf \emptyset:=\infty$. Similarly, for any non-negative increasing function $f$ on $[0, \infty)$, let

$$
f^{-1}(r):=\inf \{s>0: f(s) \geq r\}, \quad r \geq 0 .
$$

In the following four subsections, we first observe the equivalence of an $L^{1}$ functional inequality and the corresponding isoperimetric inequality, then investigate the link between the 
super Poincaré and isoperimetric inequalities, and finally extend the main results to the case with killing. For notational simplicity, in this section we set $J(\mathrm{~d} x, \mathrm{~d} y)=J(x, y) \mu(\mathrm{d} x) \mu(\mathrm{d} y)$, and $J(A, B)=\int_{A \times B} J(x, y) \mu(\mathrm{d} x) \mu(\mathrm{d} y)$ for any $A, B \subset E$.

\section{1 $\quad L^{1}$ functional and isoperimetric inequalities}

Consider the $L^{1}$ Orlicz-Sobolev inequality

$$
\|f\|_{N} \leq C \int_{E \times E}|f(x)-f(y)| J(\mathrm{~d} x, \mathrm{~d} y), \quad f \in L_{N}(\mu)
$$

and the $L^{1}$ Poincaré type inequality

$$
\|f\|_{2}^{2} \leq C_{1} \int_{E \times E}\left|f^{2}(x)-f^{2}(y)\right| J(\mathrm{~d} x, \mathrm{~d} y)+C_{2}\|f\|_{1}^{2}, \quad f \in L^{2}(\mu),
$$

where $N$ is a Young function and $C, C_{1}, C_{2}>0$ are constants. The following result provides their equivalent isoperimetric inequalities.

Theorem 2.1. (1) The inequality (2.3) implies

$$
\inf _{\mu(A) \in(0, \infty)}\left\{N^{-1}\left(\mu(A)^{-1}\right) J\left(A \times A^{c}\right)\right\} \geq \kappa
$$

holds for $\kappa=\frac{1}{2 C}$. On the other hand, if $N_{-}^{\prime}(s)>0$ for $s>0$ such that

$$
c_{N}:=\inf _{s>0} \frac{N(s)}{s N_{-}^{\prime}(s)}>0,
$$

then (2.5) implies (2.3) for $C=\frac{1}{2 c_{N} \kappa}$.

(2) The inequality (2.4) implies

$$
\mu(A) \leq 2 C_{1} J\left(A \times A^{c}\right)+\tilde{C}_{2} \mu(A)^{2}, \quad \mu(A) \in(0, \infty)
$$

for $\tilde{C}_{2}=C_{2}$. On the other hand, (2.6) implies (2.4) for $C_{2}=2 \tilde{C}_{2}$.

Proof. (1) For $A \subset E$ with $\mu(A) \in(0, \infty)$, let $f=\mathbf{1}_{A}$. Then

$$
\int_{E \times E}|f(x)-f(y)| J(\mathrm{~d} x, \mathrm{~d} y)=2 J\left(A \times A^{c}\right) .
$$

Moreover, for any $r>0$, by the definition of $N^{-1}$ we see that

$$
\int_{E} N(|f(x)| / r) \mu(\mathrm{d} x)=N\left(r^{-1}\right) \mu(A) \leq 1
$$

implies $r \geq N^{-1}\left(\mu(A)^{-1}\right)$. Therefore, $\|f\|_{N} \geq N^{-1}\left(\mu(A)^{-1}\right)$. Combining this with (2.3) and (2.7), we prove $(2.5)$ for $\kappa=\frac{1}{2 C}$. 
On the other hand, let $c_{N}>0$ and (2.5) hold. It suffices to prove (2.3) for $C=\frac{1}{2 c_{N} \kappa}$ and any $f \geq 0$ with $\|f\|_{N}=1$. By Fubini's theorem and (2.5), we have

$$
\begin{aligned}
& \int_{E \times E}|f(x)-f(y)| J(\mathrm{~d} x, \mathrm{~d} y)=2 \int_{\{f(x)>f(y)\}}\left(\int_{f(y)}^{f(x)} \mathrm{d} r\right) J(\mathrm{~d} x, \mathrm{~d} y) \\
& =2 \int_{0}^{\infty} J(\{(x, y): f(x)>r \geq f(y)\}) \mathrm{d} r \geq 2 \kappa \int_{0}^{\infty} \frac{\mathrm{d} r}{N^{-1}\left(\mu(f>r)^{-1}\right)} \\
& =2 \kappa \int_{0}^{\infty} \frac{\mu(N(f)>N(r))}{\mu(N(f)>N(r)) N^{-1}\left(\mu(N(f)>N(r))^{-1}\right)} \mathrm{d} r \\
& =2 \kappa \int_{0}^{\infty} \frac{\mu(N(f)>s)}{N_{-}^{\prime}\left(N^{-1}(s)\right) \mu(N(f)>s) N^{-1}\left(\mu(N(f)>s)^{-1}\right)} \mathrm{d} s .
\end{aligned}
$$

Since $f \geq 0$ and $\|f\|_{N}=1$, we have

$$
\mu(N(f)>s) \leq \frac{\mu(N(f))}{s}=\frac{1}{s}, \quad s>0 .
$$

Noting that $N_{-}^{\prime} \circ N^{-1}$ is increasing, by letting $t=N^{-1}\left(\mu(N(f)>s)^{-1}\right)$ we obtain

$$
\begin{aligned}
& N_{-}^{\prime}\left(N^{-1}(s)\right) \mu(N(f)>s) N^{-1}\left(\mu(N(f)>s)^{-1}\right) \\
& \leq N_{-}^{\prime}\left(N^{-1}\left(\mu(N(f)>s)^{-1}\right)\right) \mu(N(f)>s) N^{-1}\left(\mu(N(f)>s)^{-1}\right) \\
& =\frac{N_{-}^{\prime}(t) t}{N(t)} \leq \frac{1}{c_{N}}
\end{aligned}
$$

Substituting into (2.8) and noting that $\|f\|_{N}=1$, we arrive at

$$
\int_{E \times E}|f(x)-f(y)| J(\mathrm{~d} x, \mathrm{~d} y) \geq 2 \kappa c_{N} \mu(N(f))=2 \kappa c_{N}=2 \kappa c_{N}\|f\|_{N}
$$

Thus, (2.3) holds for $C=\frac{1}{2 \kappa c_{N}}$.

(2) As in (1), by applying (2.4) to $f=\mathbf{1}_{A}$ we prove (2.6) for $\tilde{C}_{2}=C_{2}$. On the other hand, let $f \in L^{2}(\mu)$ with $\|f\|_{1}=1$. Then $\mu\left(f^{2}>s\right) \leq s^{-1 / 2}$, so that, as in (2.8), (2.6) yields

$$
\begin{aligned}
& C_{1} \int_{E \times E}\left|f^{2}(x)-f^{2}(y)\right| J(\mathrm{~d} x, \mathrm{~d} y)=2 C_{1} \int_{0}^{\infty} J\left(\left\{f^{2}>s\right\} \times\left\{f^{2} \leq s\right\}\right) \mathrm{d} s \\
& \geq \int_{0}^{\infty}\left\{\mu\left(f^{2}>s\right)-\tilde{C}_{2} \mu\left(f^{2}>s\right)^{2}\right\} \mathrm{d} s \geq \mu\left(f^{2}\right)-\tilde{C}_{2} \int_{0}^{\infty} \frac{1}{\sqrt{s}} \mu(|f|>\sqrt{s}) \mathrm{d} s \\
& =\mu\left(f^{2}\right)-2 \tilde{C}_{2} \int_{0}^{\infty} \mu(|f|>\sqrt{s}) \mathrm{d} \sqrt{s}=\mu\left(f^{2}\right)-2 \tilde{C}_{2} \mu(|f|)=\mu\left(f^{2}\right)-2 \tilde{C}_{2} \mu(|f|)^{2} .
\end{aligned}
$$

Therefore, (2.6) implies (2.4) for $C_{2}=2 \tilde{C}_{2}$. 


\subsection{From super Poincaré to isoperimetric}

Let $P_{t}$ be the (sub-) Markov semigroup associated with the symmetric Dirichlet form $(\mathscr{E}, \mathscr{D}(\mathscr{E}))$.

Theorem 2.2. Assume that (2.2) holds with $\beta(\infty):=\lim _{r \rightarrow \infty} \beta(r)=0$. Let $\gamma: E \times E \rightarrow$ $[0, \infty)$ with $\gamma(x, y)>0$ and $\gamma(x, y)=\gamma(y, x)$ for $x \neq y$, and define

$$
\theta_{\gamma}(t):=\sup _{\|g\|_{\infty} \leq 1} \operatorname{ess}_{\mu \times \mu} \sup _{x \neq y} \frac{\left|P_{t} g(x)-P_{t} g(y)\right|}{\gamma(x, y)}, \quad t>0 .
$$

If

$$
\Phi_{\gamma}(s):=\int_{0}^{s} \mathrm{~d} r \int_{0}^{\beta^{-1}(r)} \theta_{\gamma}(t) \mathrm{d} t<\infty, \quad s>0
$$

then $N_{\gamma}:=\Phi_{\gamma}^{-1}$ is a Young function, and there exists a constant $C>0$ such that

$$
\|f\|_{N_{\gamma}} \leq C \int_{E \times E}|f(x)-f(y)| \gamma(x, y) J(\mathrm{~d} x, \mathrm{~d} y), \quad f \in L_{N_{\gamma}}(\mu) .
$$

To prove this result, we consider the symmetric measure

$$
J_{\gamma}(\mathrm{d} x, \mathrm{~d} y):=\gamma(x, y) J(\mathrm{~d} x, \mathrm{~d} y)
$$

on $E \times E$, and introduce the isoperimetric constants

$$
\kappa_{\gamma}(s):=\inf \left\{\frac{J_{\gamma}\left(A \times A^{c}\right)}{\mu(A)}: \mu(A) \in(0, s)\right\}, \quad s>0,
$$

where $\inf \emptyset:=\infty$. We have the following result.

Lemma 2.3. For any increasing function $G:[0, \infty) \rightarrow[0, \infty)$ with $G(0)=0$ and $G(s)>0$ for $s>0$, it holds that

$$
\int_{E} \mathrm{~d} \mu \int_{0}^{|f|} \kappa_{\gamma}\left(G(s)^{-1}\right) \mathrm{d} s \leq \frac{1}{2} \int_{E \times E}|f(x)-f(y)| J_{\gamma}(\mathrm{d} x, \mathrm{~d} y), \quad \mu(G(|f|))=1 .
$$

Consequently:

(1) If $\kappa_{\gamma}(s)>0$ for some $s>0$, then

$$
\|f\|_{2}^{2} \leq \frac{1}{2 \kappa_{\gamma}(s)} \int_{E \times E}\left|f^{2}(x)-f^{2}(y)\right| J_{\gamma}(\mathrm{d} x, \mathrm{~d} y)+\frac{2}{s}\|f\|_{1}^{2}, \quad f \in L^{2}(\mu) .
$$

(2) If $\kappa_{\gamma}(s)>0$ for all $s>0$ such that

$$
\Phi(t):=\int_{0}^{t} \frac{\mathrm{d} r}{\kappa_{\gamma}\left(r^{-1}\right)}<\infty, \quad t>0,
$$

then $N:=\Phi^{-1}$ is a Young function, and

$$
\|f\|_{N} \leq \frac{1}{2} \int_{E \times E}|f(x)-f(y)| J_{\gamma}(\mathrm{d} x, \mathrm{~d} y), \quad f \in L_{N}(\mu) .
$$


Proof. For any $f \in \mathscr{B}(E)$ with $\mu(G(|f|))=1$, we have

$$
\mu(|f|>s) \leq G(s)^{-1}, \quad s>0 .
$$

As in (2.8), this and the definition of $\kappa_{\gamma}(s)$ imply that

$$
\begin{aligned}
& \frac{1}{2} \int_{E \times E}|f(x)-f(y)| J_{\gamma}(\mathrm{d} x, \mathrm{~d} y) \geq \frac{1}{2} \int_{E \times E}|| f|(x)-| f|(y)| J_{\gamma}(\mathrm{d} x, \mathrm{~d} y) \\
& =\int_{0}^{\infty} J_{\gamma}(\{|f|>u\} \times\{|f| \leq u\}) \mathrm{d} u \geq \int_{0}^{\infty} \kappa_{\gamma}\left(G(u)^{-1}\right) \mu(|f|>u) \mathrm{d} u \\
& =\int_{E} \mathrm{~d} \mu \int_{0}^{|f|} \kappa_{\gamma}\left(G(u)^{-1}\right) \mathrm{d} u .
\end{aligned}
$$

We have proved (2.11). Below we prove assertions (1) and (2) respectively.

Assertion (1). For any $f \in \mathscr{B}(E)$ with $\|f\|_{1}=1$, we have

$$
\mu(|f|>s) \leq \frac{1}{s}, \quad s>0 .
$$

Since $\kappa_{\gamma}(s)$ is decreasing in $s$, applying $(2.11)$ to $f^{2}$ with $G(s)=s^{1 / 2}$, we derive

$$
\begin{aligned}
& \frac{1}{2} \int_{E \times E}\left|f^{2}(x)-f^{2}(y)\right| J_{\gamma}(\mathrm{d} x, \mathrm{~d} y) \geq \int_{E} \mathrm{~d} \mu \int_{0}^{f^{2}} \kappa_{\gamma}\left(u^{-1 / 2}\right) \mathrm{d} u \\
& =\int_{0}^{\infty} \kappa_{\gamma}\left(u^{-1 / 2}\right) \mu\left(f^{2}>u\right) \mathrm{d} u \geq \kappa_{\gamma}(s) \int_{s^{-2}}^{\infty} \mu\left(f^{2}>u\right) \mathrm{d} u, \quad s>0 .
\end{aligned}
$$

On the other hand, by (2.14) we have

$$
\int_{0}^{s^{-2}} \mu\left(f^{2}>u\right) \mathrm{d} u \leq \int_{0}^{s^{-2}} \frac{1}{\sqrt{u}} \mathrm{~d} u=\frac{2}{s}, \quad s>0 .
$$

Combining this with $(2.15)$ and

$$
\|f\|_{2}^{2}=\int_{0}^{\infty} \mu\left(f^{2}>u\right) \mathrm{d} u
$$

we prove (2.12).

Assertion (2). Let $N=\Phi^{-1}$. Then $N$ satisfies $N(0)=0$, and solves the equation

$$
\frac{\mathrm{d} N(s)}{\mathrm{d} s}=\kappa_{\gamma}\left(N(s)^{-1}\right) \quad \text { a.e. } s>0
$$

where $\frac{\mathrm{d} N(s)}{\mathrm{d} s}$ denotes the Radon-Nikodym derivative of $N$ with respect to the Lebesgue measure. Since $\kappa_{\gamma}(s)$ is strictly positive and decreasing in $s$, and since $\Phi(t)<\infty$ for $t>0$, it is easy to deduce from (2.16) that $N$ is a Young function, and

$$
\int_{0}^{|f|} \kappa_{\gamma}\left(N(s)^{-1}\right) \mathrm{d} s=\int_{0}^{|f|} \frac{\mathrm{d} N(s)}{\mathrm{d} s} \mathrm{~d} s=N(|f|) .
$$


Combining this with (2.11) leads to

$$
1 \leq \frac{1}{2} \int_{E \times E}|f(x)-f(y)| J_{\gamma}(\mathrm{d} x, \mathrm{~d} y), \quad \mu(N(|f|))=1,
$$

which in turn implies (2.13).

According to Lemma 2.3, for the proof of Theorem 2.2 we only need to estimate the isoperimetric constants $\kappa_{\gamma}(s)$ using (2.2). The following result can be regarded as an extension of a result of [11] (see also [31]) to non-local forms.

Lemma 2.4. Let $\gamma$ and $\theta_{\gamma}(t)$ be in Theorem 2.2. If

$$
\Theta_{\gamma}(t):=\int_{0}^{t} \theta_{\gamma}(s) \mathrm{d} s<\infty, \quad t \geq 0,
$$

then the super Poincaré inequality (2.2) implies

$$
\kappa_{\gamma}(s) \geq \frac{1-\mathrm{e}^{-1}}{2 \Theta_{\gamma}\left(\beta^{-1}(1 /(2 s))\right)}, \quad s>0 .
$$

Proof. For any $f, g \in \mathscr{D}(\mathscr{E})$, we have

$$
\begin{aligned}
\mu\left(g\left(f-P_{t} f\right)\right) & =\mu\left(f\left(g-P_{t} g\right)\right)=\int_{0}^{t} \mathscr{E}\left(f, P_{s} g\right) \mathrm{d} s \\
& =\frac{1}{2} \int_{0}^{t} \mathrm{~d} s \int_{E \times E}\left(P_{s} g(x)-P_{s} g(y)\right)(f(x)-f(y)) J(\mathrm{~d} x, \mathrm{~d} y) \\
& \leq \frac{\|g\|_{\infty}}{2}\left(\int_{0}^{t} \theta_{\gamma}(s) \mathrm{d} s\right) \int_{E \times E}|f(y)-f(x)| J_{\gamma}(\mathrm{d} x, \mathrm{~d} y) \\
& =\frac{\Theta_{\gamma}(t)\|g\|_{\infty}}{2} \int_{E \times E}|f(y)-f(x)| J_{\gamma}(\mathrm{d} x, \mathrm{~d} y) .
\end{aligned}
$$

Thus,

$$
\mu\left(\left|f-P_{t} f\right|\right) \leq \frac{\Theta_{\gamma}(t)}{2} \int_{E \times E}|f(y)-f(x)| J_{\gamma}(\mathrm{d} x, \mathrm{~d} y) .
$$

Next, by $[51,(3.4)]$, the super Poincaré inequality (2.2) is equivalent to

$$
\left\|P_{t} f\right\|_{2}^{2} \leq\|f\|_{2}^{2} \exp (-2 t / r)+\beta(r)\|f\|_{1}^{2}(1-\exp (-2 t / r)), \quad t, r>0 .
$$

In particular, for any $A \subset E$ with $\mu(A)<\infty$, we have

$$
\left\|P_{t / 2} \mathbf{1}_{A}\right\|_{2}^{2} \leq \mu(A) \exp (-t / r)+\mu(A)^{2} \beta(r)(1-\exp (-t / r)), \quad t, r>0 .
$$

Now, for $s>0$ and $A \subset E$ with $0<\mu(A)<s$, (2.18) gives

$$
\mu\left(\left|\mathbf{1}_{A}-P_{t} \mathbf{1}_{A}\right|\right) \leq \frac{\Theta_{\gamma}(t)}{2} \int_{E \times E}\left|\mathbf{1}_{A}(y)-\mathbf{1}_{A}(x)\right| J_{\gamma}(\mathrm{d} x, \mathrm{~d} y)=\Theta_{\gamma}(t) J_{\gamma}\left(A \times A^{c}\right) .
$$


On the other hand, we have

$$
\begin{aligned}
\mu\left(\left|\mathbf{1}_{A}-P_{t} \mathbf{1}_{A}\right|\right) & =\int_{A}\left(1-P_{t} \mathbf{1}_{A}\right) \mathrm{d} \mu+\int_{A^{c}} P_{t} \mathbf{1}_{A} \mathrm{~d} \mu \\
& \geq \int_{A}\left(1-P_{t} \mathbf{1}_{A}\right) \mathrm{d} \mu=\mu(A)-\int_{A} P_{t} \mathbf{1}_{A} \mathrm{~d} \mu=\mu(A)-\left\|P_{t / 2} \mathbf{1}_{A}\right\|_{2}^{2} .
\end{aligned}
$$

This together with (2.19) yields that for any $t, r>0$,

$$
\mu\left(\left|\mathbf{1}_{A}-P_{t} \mathbf{1}_{A}\right|\right) \geq \mu(A)(1-\mu(A) \beta(r))(1-\exp (-t / r)) .
$$

Taking $r=t=\beta^{-1}(1 /(2 \mu(A)))$ in the inequality above, we get that

$$
\mu\left(\left|\mathbf{1}_{A}-P_{t} \mathbf{1}_{A}\right|\right) \geq \frac{1-\mathrm{e}^{-1}}{2} \mu(A) .
$$

Combining this with $(2.20)$ we arrive at

$$
\frac{J_{\gamma}\left(A \times A^{c}\right)}{\mu(A)} \geq \frac{1-\mathrm{e}^{-1}}{2 \Theta_{\gamma}\left(\beta^{-1}(1 /(2 \mu(A)))\right)} \geq \frac{1-\mathrm{e}^{-1}}{2 \Theta_{\gamma}\left(\beta^{-1}(1 /(2 s))\right)},
$$

where in the last inequality we have used the facts that $0<\mu(A)<s, \beta$ is decreasing and $\Theta_{\gamma}$ is increasing. Therefore, (2.17) holds.

Proof of Theorem 2.2. Let $\Phi_{\gamma}$ be in Theorem 2.2. Since $\beta(s)$ is strictly positive and deceasing on $(0, \infty)$, it is easy to see that $N_{\gamma}:=\Phi_{\gamma}^{-1}$ is a Young function. Since $\beta(\infty)=0$, by Lemma 2.4 we have $\kappa_{\gamma}(s)>0$ for all $s>0$, and

$$
\begin{aligned}
\Phi(t): & =\int_{0}^{t} \frac{\mathrm{d} u}{\kappa_{\gamma}\left(u^{-1}\right)} \leq \frac{2}{1-e^{-1}} \int_{0}^{t} \Theta_{\gamma}\left(\beta^{-1}(u / 2)\right) \mathrm{d} u \\
& \leq \frac{4}{1-\mathrm{e}^{-1}} \int_{0}^{t} \Theta_{\gamma}\left(\beta^{-1}(r)\right) \mathrm{d} r=\frac{4}{1-\mathrm{e}^{-1}} \Phi_{\gamma}(t), \quad t \geq 0 .
\end{aligned}
$$

Thus, $N_{\gamma}(s):=\Phi_{\gamma}^{-1}(s) \leq \Phi^{-1}\left(4 s /\left(1-\mathrm{e}^{-1}\right)\right):=N\left(4 s /\left(1-\mathrm{e}^{-1}\right)\right)$. Combining this with $(2.13)$ and (1.6), we prove (2.9).

\subsection{From isoperimetric to super Poincaré}

Let $(\mathscr{E}, \mathscr{D}(\mathscr{E}))$ be given by (2.1). For a non-negative symmetric function $\gamma$ on $E \times E$, let $J_{\gamma}(\mathrm{d} x, \mathrm{~d} y):=\gamma(x, y) J(\mathrm{~d} x, \mathrm{~d} y)$, and $\kappa_{\gamma}(s)$ be the isoperimetric constant defined by $(2.10)$. For a Young function $N$, we aim to deduce the super Poincaré inequality (2.2) from the Orlicz-Sobolev type isoperimetric inequality

$$
\|f\|_{N} \leq C \int_{E \times E}|f(x)-f(y)| J_{\gamma}(\mathrm{d} x, \mathrm{~d} y), \quad f \in L_{N}(\mu) .
$$


To this end, we also consider the Poincaré type isoperimetric inequality

$$
\|f\|_{2}^{2} \leq r \int_{E \times E}\left|f^{2}(x)-f^{2}(y)\right| J_{\gamma}(\mathrm{d} x, \mathrm{~d} y)+\beta_{1}(r)\|f\|_{1}^{2}, \quad r>0, f \in L^{2}(\mu)
$$

for some decreasing function $\beta_{1}:(0, \infty) \rightarrow(0, \infty)$.

Theorem 2.5. Assume (2.21) for some Young function $N$ such that $s \mapsto s^{-1} N(s)$ is increasing on $(0, \infty)$. Then:

(1) For any $s>0$,

$$
\kappa_{\gamma}(s) \geq \frac{1}{2 C s N^{-1}\left(s^{-1}\right)} .
$$

(2) (2.22) holds with

$$
\beta_{1}(r):=2 \inf \left\{s>0: C s^{-1} N^{-1}(s) \leq r\right\}, \quad r>0 .
$$

(3) If the density $J(x, y):=\frac{J(\mathrm{~d} x, \mathrm{~d} y)}{\mu(\mathrm{d} x) \mu(\mathrm{d} y)}$ and $\gamma$ satisfy

$$
c_{\gamma}:=\operatorname{ess}_{\mu} \sup _{x} \int_{E} \gamma(x, y)^{2} J(x, y) \mu(\mathrm{d} y)<\infty,
$$

then (2.2) holds with

$$
\beta(r):=4 \inf \left\{s>0: s^{-1} N^{-1}(s) \leq \frac{\sqrt{r}}{2 C \sqrt{2 c_{\gamma}}}\right\}, \quad r>0 .
$$

Proof. For any $s>0$ and $A \subset E$ with $\mu(A) \in(0, s)$, take $f=N^{-1}\left(\mu(A)^{-1}\right) \mathbf{1}_{A}$. Then $\|f\|_{N}=1$ and due to $(2.21)$,

$$
1 \leq 2 C J_{\gamma}\left(A \times A^{c}\right) N^{-1}\left(\mu(A)^{-1}\right) .
$$

Therefore,

$$
\kappa_{\gamma}(s) \geq \frac{1}{2 C} \inf _{r \in(0, s)} \frac{1}{r N^{-1}\left(r^{-1}\right)} .
$$

Since $s N^{-1}\left(s^{-1}\right)$ is increasing in $s>0$, this implies (1).

It is easy to see that (2) follows from (1) and Lemma 2.3(1). It remains to prove (3). By (1), Lemma 2.3(1), and the Cauchy-Schwarz inequality, we obtain

$$
\begin{aligned}
\|f\|_{2}^{2} & \leq C s N^{-1}\left(s^{-1}\right) \int_{E \times E}\left|f^{2}(x)-f^{2}(y)\right| J_{\gamma}(\mathrm{d} x, \mathrm{~d} y)+\frac{2}{s}\|f\|_{1}^{2} \\
& \leq C s N^{-1}\left(s^{-1}\right)\left(\int_{E \times E}(f(x)-f(y))^{2} J(\mathrm{~d} x, \mathrm{~d} y)\right)^{1 / 2}
\end{aligned}
$$




$$
\begin{gathered}
\times\left(\int_{E \times E}(f(x)+f(y))^{2} \gamma(x, y)^{2} J(\mathrm{~d} x, \mathrm{~d} y)\right)^{1 / 2}+\frac{2}{s}\|f\|_{1}^{2} \\
\leq 2 C \sqrt{2 c_{\gamma}} s N^{-1}\left(s^{-1}\right) \sqrt{\mathscr{E}(f, f)\|f\|_{2}^{2}}+\frac{2}{s}\|f\|_{1}^{2} \\
\leq \frac{1}{2}\|f\|_{2}^{2}+4 C^{2} c_{\gamma}\left(s N^{-1}\left(s^{-1}\right)\right)^{2} \mathscr{E}(f, f)+\frac{2}{s}\|f\|_{1}^{2}, \quad s>0,
\end{gathered}
$$

where in the third inequality we have used (2.23). This implies (2.2) for the desired $\beta$.

Similarly, we have the following result.

Theorem 2.6. Assume that (2.22) holds with $\beta_{1}(\infty):=\lim _{r \rightarrow \infty} \beta_{1}(r)=0$. Then:

(1) For any $s>0$,

$$
\kappa_{\gamma}(s) \geq \frac{1}{4 \beta_{1}^{-1}(1 /(2 s))}
$$

(2) If

$$
\Phi(t):=4 \int_{0}^{t} \beta_{1}^{-1}(r / 2) \mathrm{d} r<\infty, \quad t>0,
$$

then (2.21) holds with $N:=\Phi^{-1}$.

(3) (2.23) implies (2.2) with

$$
\beta(r):=2 \beta_{1}\left(\sqrt{r} /\left(2 \sqrt{2 c_{\gamma}}\right)\right), \quad r>0 .
$$

Proof. By Theorem 2.1(2), (2.22) implies

$$
\mu(A) \leq 2 r J_{\gamma}\left(A \times A^{c}\right)+\beta_{1}(r) \mu(A)^{2}, \quad r>0 .
$$

Thus,

$$
1 \leq 2 r \frac{J_{\gamma}\left(A \times A^{c}\right)}{\mu(A)}+\beta_{1}(r) \mu(A) \leq 2 r \frac{J_{\gamma}\left(A \times A^{c}\right)}{\mu(A)}+\beta_{1}(r) s, \quad r>0 .
$$

Taking $r=\beta_{1}^{-1}\left((2 s)^{-1}\right)$ in the inequality above, we get that

$$
\frac{J_{\gamma}\left(A \times A^{c}\right)}{\mu(A)} \geq \frac{1}{4 \beta_{1}^{-1}(1 /(2 s))} .
$$

This implies (1).

(2) immediately follows from (1) and Lemma 2.3(2), and (3) can be proved by the argument for Theorem 2.5(3).

As a consequence of Theorem 2.5(2) and Theorem 2.6(2), we have the following correspondence of (2.21) and (2.22).

Corollary 2.7. Let $p_{1}, p_{2}, p>1$ and $q \in \mathbb{R}$ be constants. Then, 
(1) (2.21) holds with $N(s)=s^{p_{1}} \wedge s^{p_{2}}$ if and only if (2.22) holds with

$$
\beta_{1}(r):=c\left(r^{-\frac{p_{1}}{p_{1}-1}} \vee r^{-\frac{p_{2}}{p_{2}-1}}\right), \quad r>0
$$

for some constant $c>0$.

(2) (2.21) holds with $N(s)=s^{p_{1}} \vee s^{p_{2}}$ if and only if (2.22) holds with

$$
\beta_{1}(r):=c\left(r^{-\frac{p_{1}}{p_{1}-1}} \wedge r^{-\frac{p_{2}}{p_{2}-1}}\right), \quad r>0
$$

for some constant $c>0$.

(3) Let $\lambda \geq 2$ such that $N(s):=s^{p}\left\{\log \left(\lambda+s^{-1}\right)\right\}^{q}$ is Young function and $s \mapsto s^{-1} N(s)$ is increasing on $(0, \infty)$. Then, (2.21) holds with $N(s)$ if and only if $(2.22)$ holds with

$$
\beta_{1}(r):=c r^{-\frac{p}{p-1}}\{\log (2+r)\}^{-\frac{q}{p-1}}, \quad r>0
$$

for some constant $c>0$.

(4) Let $\lambda \geq 2$ such that $N(s):=s^{p}\{\log (\lambda+s)\}^{q}$ is Young function and $s \mapsto s^{-1} N(s)$ is increasing on $(0, \infty)$. Then, $(2.21)$ holds with $N(s)$ if and only if $(2.22)$ holds with

$$
\beta_{1}(r):=c r^{-\frac{p}{p-1}}\left\{\log \left(2+r^{-1}\right)\right\}^{-\frac{q}{p-1}}, \quad r>0
$$

for some constant $c>0$.

\subsection{Extension to the case with killing}

We will add a potential term to the Dirichlet form $(\mathscr{E}, \mathscr{D}(\mathscr{E}))$ given in $(2.1)$. Let $V$ be a non-negative measurable function on $(E, \mathscr{F})$ such that the class

$$
\mathscr{D}\left(\mathscr{E}_{V}\right):=\left\{f \in L^{2}(\mu): \mathscr{E}_{V}(f, f):=\mathscr{E}(f, f)+\int_{E} f^{2} V(\mathrm{~d} x)<\infty\right\}
$$

is dense in $L^{2}(\mu)$, where $V(\mathrm{~d} x):=V(x) \mu(\mathrm{d} x)$. Then $\left(\mathscr{E}_{V}, \mathscr{D}\left(\mathscr{E}_{V}\right)\right)$ is a Shrödinger type symmetric energy form in $L^{2}(\mu)$, where

$$
\mathscr{E}_{V}(f, g):=\mathscr{E}(f, g)+\int_{E} f(x) g(x) V(\mathrm{~d} x), \quad f, g \in \mathscr{D}\left(\mathscr{E}_{V}\right)
$$

It is standard that by enlarging the state space we are able to reduce to present setting to the case without killing, see [30,17]. More precisely, let $\bar{E}:=E \cup\{\Delta\}$ for an additional state $\Delta$, and define

$$
\begin{aligned}
& \bar{\mu}(\mathrm{d} x)=\mathbf{1}_{E}(x) \mu(\mathrm{d} x)+\delta_{\Delta}(\mathrm{d} x), \\
& \bar{J}(\mathrm{~d} x, \mathrm{~d} y)=\mathbf{1}_{E \times E} J(\mathrm{~d} x, \mathrm{~d} y)+\mathbf{1}_{\{\Delta\} \times E}(x, y) \delta_{\Delta}(\mathrm{d} x) V(\mathrm{~d} y)+\mathbf{1}_{E \times\{\Delta\}}(x, y) V(\mathrm{~d} x) \delta_{\Delta}(\mathrm{d} y),
\end{aligned}
$$


where $\delta_{\Delta}$ is the Dirac measure at point $\Delta$. Since $J(\mathrm{~d} x, \mathrm{~d} y)=J(x, y) \mu(\mathrm{d} x) \mu(\mathrm{d} y)$, we have

$$
\bar{J}(\mathrm{~d} x, \mathrm{~d} y)=\bar{J}(x, y) \bar{\mu}(\mathrm{d} x) \bar{\mu}(\mathrm{d} y),
$$

where

$$
\bar{J}(x, y):=\mathbf{1}_{E \times E}(x, y) J(x, y)+\mathbf{1}_{\{\Delta\} \times E}(x, y) V(y)+\mathbf{1}_{E \times\{\Delta\}}(x, y) V(x) .
$$

Next, for a non-negative symmetric function $\gamma$ on $E \times E$ and a non-negative function $\xi$ on $E$, let

$$
\bar{\gamma}(x, y)=\mathbf{1}_{E \times E}(x, y) \gamma(x, y)+\mathbf{1}_{\{\Delta\} \times E}(x, y) \xi(y)+\mathbf{1}_{E \times\{\Delta\}}(x, y) \xi(x) .
$$

Then for any $x \in E$,

$$
\int_{\bar{E}} \bar{\gamma}(x, y)^{2} \bar{J}(x, y) \bar{\mu}(\mathrm{d} y)=\int_{E} \gamma(x, y)^{2} J(x, y) \mu(\mathrm{d} y)+\xi(x)^{2} V(x) .
$$

Finally, for any measurable function $f$ on $E$, we extend it into $\bar{f}$ defined on $\bar{E}$ and by letting $\bar{f}(\Delta)=0$. Then

$$
\mathscr{E}_{V}(f, g)=\overline{\mathscr{E}}(\bar{f}, \bar{g}):=\frac{1}{2} \int_{\bar{E} \times \bar{E}}(\bar{f}(x)-\bar{f}(y))(\bar{g}(x)-\bar{g}(y)) \bar{J}(\mathrm{~d} x, \mathrm{~d} y), \quad f, g \in \mathscr{D}\left(\mathscr{E}_{V}\right) .
$$

Let $P_{t}^{V}$ be the (sub)-Markov semigroup on $L^{2}(\mu)$ associated to $\left(\mathscr{E}_{V}, \mathscr{D}\left(\mathscr{E}_{V}\right)\right)$, while $\bar{P}_{t}$ is the corresponding semigroup on $L^{2}(\bar{\mu})$. We have

$$
\begin{aligned}
\bar{\theta}(t) & :=\sup _{\|\bar{g}\|_{\infty} \leq 1} \operatorname{ess}_{\bar{\mu} \times \bar{\mu}} \sup _{x \neq y} \frac{\left|\bar{P}_{t} \bar{g}(x)-\bar{P} t \bar{g}(y)\right|}{\bar{\gamma}(x, y)} \\
& =\sup _{\|g\|_{\infty} \leq 1} \operatorname{ess}_{\mu \times \mu} \sup _{x \neq y} \max \left\{\frac{\left|P_{t} g(x)-P_{t} g(y)\right|}{\gamma(x, y)}, \frac{\left|P_{t} g(x)\right|}{\xi(x)}\right\}, \quad t>0 .
\end{aligned}
$$

With the aid of all the notations above, by applying Theorem 2.2 and Theorem 2.5 to $\overline{\mathscr{E}}$ and $\bar{\mu}$ we obtain the following result.

Theorem 2.8. Suppose that the super Poincaré inequality $(2.2)$ holds for $\left(\mathscr{E}_{V}, \mathscr{D}\left(\mathscr{E}_{V}\right)\right)$ replacing $(\mathscr{E}, \mathscr{D}(\mathscr{E}))$ with some decreasing function $\beta:(0, \infty) \rightarrow(0, \infty)$ satisfying that $\beta(\infty)=0$. If

$$
\bar{\Phi}_{\gamma}(s):=\int_{0}^{s} \mathrm{~d} r \int_{0}^{\beta^{-1}(r)} \bar{\theta}(t) \mathrm{d} t<\infty, \quad s>0,
$$

then $\bar{N}_{\gamma}:=\bar{\Phi}_{\gamma}^{-1}$ is a Young function, and there exists a constant $C>0$ such that

$$
\|f\|_{\bar{N}_{\gamma}} \leq C\left(\int_{E \times E}|f(x)-f(y)| \gamma(x, y) J(\mathrm{~d} x, \mathrm{~d} y)+\int_{E}|f(x)| \xi(x) V(\mathrm{~d} x)\right)
$$

holds for all $f \in L_{\bar{N}_{\gamma}}(\mu)$. 
On the other hand, suppose that

$$
\sup _{x \in E}\left(\int_{E} \gamma(x, y)^{2} J(x, y) \mu(\mathrm{d} y)+\xi(x)^{2} V(x)\right)<\infty .
$$

If (2.26) holds for some Young function $N$ replacing $\bar{N}_{\gamma}$ and satisfying that $s \mapsto s^{-1} N(s)$ is increasing on $(0, \infty)$, then there exist constants $c_{1}, c_{2}>0$ such that $(2.2)$ holds for $\left(\mathscr{E}_{V}, \mathscr{D}\left(\mathscr{E}_{V}\right)\right)$ replacing $(\mathscr{E}, \mathscr{D}(\mathscr{E}))$ with

$$
\beta(r):=c_{1} \inf \left\{s>0: s^{-1} N^{-1}(s) \leq c_{2} \sqrt{r}\right\}, \quad r>0 .
$$

\section{Proof of Theorem 1.1 and extensions}

\subsection{Proof of Theorem 1.1}

By Theorem 2.1(1), it suffices to prove (1.7) and assertions (1) and (2). Let $E=\mathbb{R}^{n}$ and $\mu(\mathrm{d} x)$ be the Lebesgue measure. Consider the symmetric $\alpha$-stable process on $\mathbb{R}^{n}$ with jumping kernel

$$
J(x, y):=\mathbf{1}_{\{x \neq y\}}|x-y|^{-(n+\alpha)}, \quad x, y \in \mathbb{R}^{n} .
$$

Let $P_{t}$ be the Markov semigroup generated by the Dirichlet form

$$
\mathscr{E}(f, g):=\frac{1}{2} \int_{\mathbb{R}^{n} \times \mathbb{R}^{n}}(f(x)-f(y))(g(x)-g(y)) J(x, y) \mathrm{d} x \mathrm{~d} y, \quad f, g \in \mathscr{D}(\mathscr{E}) .
$$

It is well known that for some constant $c_{1} \geq 1$, we have the heat kernel upper bound (see for instance [20, Theorem 3.2])

$$
\left\|P_{t}\right\|_{L^{1}(\mu) \rightarrow L^{\infty}(\mu)} \leq \frac{c_{1}}{t^{n / \alpha}}, \quad t>0,
$$

as well as the gradient estimate (see for instance [45, Theorem 1.3 and Example 1.4])

$$
\left\|\nabla P_{t} f\right\|_{\infty}:=\sup _{x \in \mathbb{R}^{n}} \limsup _{y \rightarrow x} \frac{\left|P_{t} f(y)-P_{t} f(x)\right|}{|y-x|} \leq \frac{c_{1}}{t^{1 / \alpha}}\|f\|_{\infty}, \quad f \in L^{\infty}(\mu), t>0 .
$$

The heat kernel upper bound (3.1) is equivalent to the Sobolev/Nash inequality with dimension $2 n / \alpha$ (see [22] or [20, Section 3]), or the super Poincaré inequality (2.2) with

$$
\beta(r)=c_{2} r^{-n / \alpha}, \quad r>0
$$

for some constant $c_{2} \geq 1$, see [51, 52] or [54].

Now, for any $h$ satisfying conditions (i) and (ii), the gradient estimate (3.2) yields

$$
\begin{aligned}
\theta(t) & :=\sup _{\|g\|_{\infty} \leq 1} \operatorname{ess}_{\mu \times \mu} \sup _{x \neq y} \frac{\left|P_{t} g(x)-P_{t} g(y)\right|}{h(|x-y|)} \leq \sup _{s>0} \frac{2 c_{1}}{h(s)}\left(1 \wedge \frac{s}{t^{1 / \alpha}}\right) \\
& =2 c_{1} \sup _{s>0}\left(\frac{1}{h(s)} \wedge \frac{s}{h(s) t^{1 / \alpha}}\right)=\frac{2 c_{1}}{h\left(t^{1 / \alpha}\right)}, \quad t>0,
\end{aligned}
$$


where the last step follows from the fact that $h(s)^{-1}$ is decreasing while $\operatorname{sh}(s)^{-1}$ is increasing so that the sup is reached at $s=t^{1 / \alpha}$ which solves $\frac{1}{h(s)}=\frac{s}{h(s) t^{1 / \alpha}}$.

Finally, let $\gamma(x, y)=h(|x-y|)$. By (3.3) and (3.4), we have

$$
\Phi_{\gamma}(s):=\int_{0}^{s} \mathrm{~d} r \int_{0}^{\beta^{-1}(r)} \theta(t) \mathrm{d} t \leq 2 c_{1} \int_{0}^{s} \mathrm{~d} r \int_{0}^{\left(r / c_{2}\right)^{-\alpha / n}} \frac{\mathrm{d} t}{h\left(t^{1 / \alpha}\right)} \leq c_{3} \Phi_{h}\left(c_{4} s\right), \quad s \geq 0
$$

for some constants $c_{3}, c_{4} \geq 1$. Therefore, by Theorem 2.2 and the property (1.6), we prove (1.7) for some constant $C>0$.

Below we verify (1.9)-(1.12) and their sharpness respectively.

(a) For (1.9). Let $\alpha=1$ and $h(s)=s^{1-\alpha_{1} / 2} \wedge s^{1-\alpha_{2} / 2}$ for $s \geq 0$. Then

$$
\Phi_{h}(s):=\int_{0}^{s} \mathrm{~d} t \int_{0}^{t^{-1 / n}} \frac{\mathrm{d} r}{h(r)} \leq c_{5}\left(s^{\frac{n-\alpha_{1} / 2}{n}} \vee s^{\frac{n-\alpha_{2} / 2}{n}}\right), \quad s \geq 0
$$

holds for some constant $c_{5}>0$. So,

$$
N_{h}:=\Phi_{h}^{-1} \geq c_{6} N_{\alpha_{1}, \alpha_{2}}^{\wedge}
$$

holds for some constant $c_{6}>0$. Therefore, (1.9) follows from (1.7).

To verify the sharpness of (1.9), let $N$ be a Young function such that $N \npreceq N_{\alpha_{1}, \alpha_{2}}^{\wedge}$. We have

$$
\left(\limsup _{s \downarrow 0}+\limsup _{s \uparrow \infty}\right) \frac{N(s)}{N_{\alpha_{1}, \alpha_{2}}^{\wedge}(s)}=\infty
$$

Let

$$
f_{s}(x):=(s-|x|)^{+}, \quad s>0, x \in \mathbb{R}^{n} .
$$

Then

$$
\left|f_{s}(x)-f_{s}(y)\right| \leq(s \wedge|x-y|)\left\{\mathbf{1}_{B(0, s)}(x)+\mathbf{1}_{B(0, s)}(y)\right\} .
$$

Thus, there exist constants $c_{7}, c_{8}>0$ such that

$$
\begin{aligned}
& \int_{\mathbb{R}^{n} \times \mathbb{R}^{n}} \frac{\left|f_{s}(x)-f_{s}(y)\right|}{|x-y|^{n+\alpha_{1} / 2} \vee|x-y|^{n+\alpha_{2} / 2}} \mathrm{~d} x \mathrm{~d} y \\
& \leq 2 \int_{B(0, s)} \mathrm{d} x \int_{\mathbb{R}^{n}} \frac{s \wedge|x-y|}{|x-y|^{n+\alpha_{1} / 2} \vee|x-y|^{n+\alpha_{2} / 2}} \mathrm{~d} y \\
& \leq c_{7} \int_{B(0, s)}\left(s^{1-\alpha_{1} / 2} \wedge s^{1-\alpha_{2} / 2}\right) \mathrm{d} x \leq c_{8}\left(s^{n+1-\alpha_{1} / 2} \wedge s^{n+1-\alpha_{2} / 2}\right), \quad s \geq 0 .
\end{aligned}
$$

If (1.9) holds for $N$ replacing $N_{\alpha_{1}, \alpha_{2}}^{\wedge}$, then

$$
\left\|f_{s}\right\|_{N} \leq c_{0}\left(s^{n+1-\alpha_{1} / 2} \wedge s^{n+1-\alpha_{2} / 2}\right), \quad s>0
$$


holds for some constant $c_{0}>0$. Therefore, there exist constants $c_{9}, c_{10}>0$ such that

$$
\begin{aligned}
1 & \geq \int_{\mathbb{R}^{n}} N\left(\left\{c_{0}\left(s^{n+1-\alpha_{1} / 2} \wedge s^{n+1-\alpha_{2} / 2}\right)\right\}^{-1} f_{s}(x)\right) \mathrm{d} x \\
& \geq \int_{\{s / 4 \leq|x| \leq 3 s / 4\}} N\left(c_{9}\left(s^{\alpha_{1} / 2-n} \vee s^{\alpha_{2} / 2-n}\right)\right) \mathrm{d} x \\
& \geq c_{10} s^{n} N\left(c_{9}\left(s^{\alpha_{1} / 2-n} \vee s^{\alpha_{2} / 2-n}\right)\right), \quad s>0 .
\end{aligned}
$$

Noting that

$$
\inf _{s>0} s^{n} N_{\alpha_{1}, \alpha_{2}}^{\wedge}\left(c_{9}\left(s^{\alpha_{1} / 2-n} \vee s^{\alpha_{2} / 2-n}\right)\right)>0,
$$

from (3.5) and (3.7) we conclude that

$$
1 \geq\left(\limsup _{s \downarrow 0}+\limsup _{s \uparrow \infty}\right) c_{10} s^{n} N\left(c_{9}\left(s^{\alpha_{1} / 2-n} \vee s^{\alpha_{2} / 2-n}\right)\right)=\infty,
$$

which is impossible.

(b) For (1.10). Let $\alpha=1$ and $h(s)=s^{1-\alpha_{1} / 2} \vee s^{1-\alpha_{2} / 2}$ for $s \geq 0$. Then

$$
\Phi_{h}(s):=\int_{0}^{s} \mathrm{~d} t \int_{0}^{t^{-1 / n}} \frac{\mathrm{d} r}{h(r)} \leq c_{5}^{\prime}\left(s^{\frac{n-\alpha_{1} / 2}{n}} \wedge s^{\frac{n-\alpha_{2} / 2}{n}}\right), \quad s \geq 0
$$

holds for some constant $c_{5}^{\prime}>0$. So,

$$
N_{h}:=\Phi_{h}^{-1} \geq c_{6}^{\prime} N_{\alpha_{1}, \alpha_{2}}^{\vee}
$$

holds for some constant $c_{6}^{\prime}>0$. Therefore, (1.10) follows from (1.7).

As in (a), we have

$$
\int_{\mathbb{R}^{n} \times \mathbb{R}^{n}} \frac{\left|f_{s}(x)-f_{s}(y)\right|}{|x-y|^{n+\alpha_{1} / 2} \wedge|x-y|^{n+\alpha_{2} / 2}} \mathrm{~d} x \mathrm{~d} y \leq c_{7}^{\prime}\left(s^{n+1-\alpha_{1} / 2} \vee s^{n+1-\alpha_{2} / 2}\right), \quad s>0 .
$$

for some constant $c_{7}^{\prime}>0$. Moreover, for any Young function $N$ with $N \npreceq N_{\alpha_{1}, \alpha_{2}}^{\wedge}$, and for any constant $c_{0}>0$, we have

$$
\left(\limsup _{s \downarrow 0}+\limsup _{s \uparrow \infty}\right) \int_{\mathbb{R}^{n}} N\left(\left\{c_{0}\left(s^{n+1-\alpha_{1} / 2} \vee s^{n+1-\alpha_{2} / 2}\right)\right\}^{-1} f_{s}(x)\right) \mathrm{d} x=\infty,
$$

so that (1.10) does not hold for $N$ replacing $N_{\alpha_{1}, \alpha_{2}}^{\vee}$.

(c) For (1.11). Let $\lambda_{0} \geq 2$ such that

$$
h(s):=s^{\alpha / 2}\left\{\log \left(\lambda_{0}+s^{-1}\right)\right\}^{q}, \quad s \geq 0
$$

satisfies condition (i). Then there exists a constant $c_{11}>0$ such that

$$
\Phi_{h}(s):=\int_{0}^{s} \mathrm{~d} t \int_{0}^{t^{-\frac{1}{n}}} \frac{r^{(\alpha / 2)-1}}{\left\{\log \left(\lambda_{0}+r^{-1}\right)\right\}^{q}} \mathrm{~d} r \leq \frac{c_{11} s^{\frac{n-\alpha / 2}{n}}}{\{\log (2+s)\}^{q}}, \quad s \geq 0 .
$$


Thus,

$$
N_{h}:=\Phi_{h}^{-1} \geq c_{12} N_{\alpha}^{\log , q,+}
$$

holds for some constant $c_{12}>0$. Therefore, (1.11) follows from (1.7). The sharpness can be verified with reference functions $f_{s}$ as above.

(d) For (1.12). We take

$$
h(s):=s^{\alpha / 2}\left\{\log \left(\lambda_{0}+s\right)\right\}^{p}, \quad s \geq 0
$$

for some $\lambda_{0} \geq 2$ large enough such that condition (i) is satisfied. Then there is a constant $c_{11}^{\prime}>0$ such that

$$
\Phi_{h}(s):=\int_{0}^{s} \mathrm{~d} t \int_{0}^{t^{-\frac{1}{n}}} \frac{r^{(\alpha / 2)-1}}{\left\{\log \left(\lambda_{0}+r\right)\right\}^{p}} \mathrm{~d} r \leq \frac{c_{11}^{\prime} s^{\frac{n-\alpha / 2}{n}}}{\left\{\log \left(2+s^{-1}\right)\right\}^{p}}, \quad s \geq 0 .
$$

Hence,

$$
N_{h}:=\Phi_{h}^{-1} \geq c_{12}^{\prime} N_{\alpha}^{l o g, p,-}
$$

holds for some constant $c_{12}^{\prime}>0$. Therefore, from (1.7) we can get (1.12). Similar to (c), one can verify the sharpness of (1.12) by using reference functions $f_{s}$ as above.

\subsection{Extension to the truncated $\alpha$-stable form}

Theorem 3.1. Let $n \geq 2, \alpha \in(0,2)$, and let $h:(0, \infty) \rightarrow(0, \infty)$ satisfy condition (i) in Theorem 1.1 and

$$
\tilde{\Phi}_{h}(s):=\int_{0}^{s} \mathrm{~d} t \int_{0}^{t^{-\frac{\alpha}{n}} \vee t^{-\frac{2}{n}}} \frac{\mathrm{d} r}{h\left(r^{\frac{1}{\alpha}} \wedge r^{\frac{1}{2}}\right)}<\infty, \quad s \geq 0 .
$$

Let $\tilde{N}_{h}=\tilde{\Phi}_{h}^{-1}$. Then there exists a constant $C>0$ such that

$$
\|f\|_{\tilde{N}_{h}} \leq C \int_{\{|x-y| \leq 1\}}|f(x)-f(y)| \frac{h(|x-y|)}{|x-y|^{n+\alpha}} \mathrm{d} x \mathrm{~d} y, \quad f \in L_{\tilde{N}_{h}}\left(\mathbb{R}^{n}\right) .
$$

Consequently, for $\tilde{N}_{\alpha}(s):=s^{\frac{n}{n-\alpha / 2}} \wedge s^{\frac{n}{n-1}}$, there exists a constant $C>0$ such that

$$
\|f\|_{\tilde{N}_{\alpha}} \leq C \int_{\{|x-y| \leq 1\}} \frac{|f(x)-f(y)|}{|x-y|^{n+\alpha / 2}} \mathrm{~d} x \mathrm{~d} y, \quad f \in L_{\tilde{N}_{\alpha}}\left(\mathbb{R}^{n}\right) .
$$

This inequality fails if $\tilde{N}_{\alpha}$ is replaced by a Young function $N \npreceq \tilde{N}_{\alpha}$. 
Proof. Consider the following truncated $\alpha$-stable Dirichlet form

$$
\mathscr{E}(f, g):=\frac{1}{2} \int_{\{|x-y| \leq 1\}}(f(x)-f(y))(g(x)-g(y)) \frac{1}{|x-y|^{d+\alpha}} \mathrm{d} x \mathrm{~d} y, \quad f, g \in \mathscr{D}(\mathscr{E}) .
$$

Let $P_{t}$ be the associated Markov semigroup. Then, by [19, Proposition 2.2] and [45, Theorem 1.3 and Example 1.5], we have

$$
\left\|P_{t}\right\|_{L^{1}(\mu) \rightarrow L^{\infty}(\mu)} \leq \frac{c_{1}}{t^{n / \alpha} \wedge t^{n / 2}}, \quad t>0
$$

and

$$
\left\|\nabla P_{t} f\right\|_{\infty} \leq \frac{c_{1}}{t^{1 / \alpha} \wedge t^{1 / 2}}\|f\|_{\infty}, \quad f \in L^{\infty}(\mu), t>0
$$

for some constant $c_{1} \geq 1$. By (3.10) and [52, Theorem 4.5], the super Poincaré inequality (2.2) holds with

$$
\beta(r)=c_{2}\left(r^{-n / \alpha} \vee r^{-n / 2}\right), \quad r>0
$$

for some constant $c_{2} \geq 1$. On the other hand, by (3.11) and the argument of (3.4), for any $h$ satisfying condition (i),

$$
\begin{aligned}
\theta(t): & =\sup _{\|g\|_{\infty} \leq 1} \operatorname{ess}_{\mu \times \mu} \sup _{x \neq y} \frac{\left|P_{t} g(x)-P_{t} g(y)\right|}{h(|x-y|)} \\
& \leq \sup _{s>0} \frac{2 c_{1}}{h(s)}\left(1 \wedge \frac{s}{t^{1 / \alpha} \wedge t^{1 / 2}}\right)=\frac{2 c_{1}}{h\left(t^{1 / \alpha} \wedge t^{1 / 2}\right)}, \quad t>0 .
\end{aligned}
$$

Thus, let $\gamma(x, y)=h(|x-y|)$. By (3.12) and (3.13), for $s>0$,

$$
\Phi_{\gamma}(s):=\int_{0}^{s} \mathrm{~d} r \int_{0}^{\beta^{-1}(r)} \theta(t) \mathrm{d} t \leq 2 c_{1} \int_{0}^{s} \mathrm{~d} r \int_{0}^{\left(r / c_{2}\right)^{-\alpha / n} \vee\left(r / c_{2}\right)^{-2 / n}} \frac{\mathrm{d} t}{h\left(t^{1 / \alpha} \wedge t^{1 / 2}\right)} \leq c_{3} \tilde{\Phi}_{h}\left(c_{4} s\right)
$$

holds with some constants $c_{3}, c_{4} \geq 1$. Therefore, by (ii') and Theorem 2.2 we prove (3.8) for some constant $C>0$.

Below we verify (3.9) and its sharpness. Let $h(s)=s^{\alpha / 2} \vee s$ for $s \geq 0$. Then

$$
\tilde{\Phi}_{h}(s):=\int_{0}^{s} \mathrm{~d} r \int_{0}^{r^{-\alpha / n} \vee r^{-2 / n}} \frac{\mathrm{d} t}{h\left(t^{1 / \alpha} \wedge t^{1 / 2}\right)} \leq c_{5}\left(s^{\frac{n-\alpha / 2}{n}} \vee s^{\frac{n-1}{n}}\right), \quad s \geq 0
$$

holds for some constant $c_{5}>0$, where in the inequality we have used the fact that $n \geq 2$. So,

$$
\tilde{N}_{h}:=\tilde{\Phi}_{h}^{-1} \geq c_{6} \tilde{N}_{\alpha}
$$

holds for some constant $c_{6}>0$. Therefore, (3.9) follows from (1.7). 
Let $f_{s}$ be the function in the argument of Theorem 1.1. Then there exists a constant $c_{7}>0$ such that

$$
\begin{aligned}
\int_{\{|x-y| \leq 1\}} \frac{\left|f_{s}(x)-f_{s}(y)\right|}{|x-y|^{n+\alpha}} \mathrm{d} x \mathrm{~d} y & \leq 2 \int_{B(0, s)} \mathrm{d} x \int_{\{|y-x| \leq 1\}} \frac{s \wedge|x-y|}{|x-y|^{n+\alpha / 2}} \mathrm{~d} y \\
& \leq c_{7}\left(s^{n+1-\alpha / 2} \wedge s^{n}\right), \quad s \geq 0 .
\end{aligned}
$$

Let $N$ be a Young function such that $N \npreceq \tilde{N}_{\alpha}$. We have

$$
\left(\limsup _{s \downarrow 0}+\limsup _{s \uparrow \infty}\right) \frac{N(s)}{\tilde{N}_{\alpha}(s)}=\infty .
$$

Suppose that (3.9) holds for $N$. Then

$$
\left\|f_{s}\right\|_{N} \leq c_{0}\left(s^{n+1-\alpha / 2} \wedge s^{n}\right), \quad s>0
$$

holds for some constant $c_{0}>0$, so that

$$
\begin{aligned}
1 & \geq \int_{\mathbb{R}^{n}} N\left(\left\{c_{0}\left(s^{n+1-\alpha / 2} \wedge s^{n}\right)\right\}^{-1} f_{s}(x)\right) \mathrm{d} x \\
& \geq \int_{\{s / 4 \leq|x| \leq 3 s / 4\}} N\left(c_{8}\left(s^{\alpha / 2-n} \vee s^{1-n}\right)\right) \mathrm{d} x \geq c_{9} s^{n} N\left(c_{8}\left(s^{\alpha / 2-n} \vee s^{1-n}\right)\right), \quad s>0 .
\end{aligned}
$$

Combining this with all the estimates above, we obtain that

$$
1 \geq\left(\limsup _{s \downarrow 0}+\limsup _{s \uparrow \infty}\right) c_{9} s^{n} N\left(c_{8}\left(s^{\alpha / 2-n} \vee s^{1-n}\right)\right)=\infty
$$

which is impossible. Therefore, (3.9) does not hold for $N$, and so we verify the sharpness of (3.9).

\subsection{Extension to discrete $\alpha$-stable Dirichlet form}

In this subsection, let $E=\mathbb{Z}^{n}$ and $\mu$ be the counting measure. Under this setting, the Orlicz norm $\|\cdot\|_{N}$ for a Young function $N$ is essentially determined by $N(s)$ for small $s>0$. In particular, for any two Young functions $N_{1}$ and $N_{2},\|\cdot\|_{N_{1}} \leq c\|\cdot\|_{N_{2}}$ for some constant $c>0$ if and only if there is a constant $c^{\prime}>0$ such that $N_{1}(s) \leq c^{\prime} N_{2}(s)$ holds for all $s \in[0,1]$. Moreover, since $|x-y| \geq 1$ for $x \neq y$, we have $2 \leq 2+|x-y|^{-1} \leq 3$, and for $\alpha_{1} \leq \alpha_{2}$,

$$
|x-y|^{n+\alpha_{1} / 2} \vee|x-y|^{n+\alpha_{2} / 2}=|x-y|^{n+\alpha_{2} / 2}, \quad|x-y|^{n+\alpha_{1} / 2} \wedge|x-y|^{n+\alpha_{2} / 2}=|x-y|^{n+\alpha_{1} / 2} .
$$

Therefore, in assertion (1) of Theorem 1.1 we will take $\alpha_{1}=\alpha_{2}=\alpha$, and in assertion (2) we only consider $N_{\alpha}^{\log , p,-}:=\left\{s \log ^{p}\left(\lambda+s^{-1}\right)\right\}^{\frac{n}{n-\alpha / 2}}$ with some constant $\lambda \geq 2$. 
Theorem 3.2. Assertions in Theorem 1.1 hold for the counting measure $\mu$ on $\mathbb{Z}^{n}$ replacing the Lebesgue measure on $\mathbb{R}^{n}$. More precisely, for any $\alpha \in(0,2)$ and $h \in \mathscr{H}_{\alpha}$, there exists a constant $C>0$ such that

$$
\|f\|_{N_{h}} \leq C \sum_{x, y \in \mathbb{Z}^{n}, x \neq y}|f(x)-f(y)| \frac{h(|x-y|)}{|x-y|^{n+\alpha}}, \quad f \in L_{N_{h}}\left(\mathbb{Z}^{n}\right) .
$$

Consequently:

(1) There exists a constant $C>0$ such that

$$
\|f\|_{\frac{n}{n-\alpha / 2}} \leq C \sum_{x, y \in \mathbb{Z}^{n}, x \neq y} \frac{|f(x)-f(y)|}{|x-y|^{n+\alpha / 2}}, \quad f \in L^{\frac{n}{n-\alpha / 2}}\left(\mathbb{Z}^{n}\right) .
$$

This inequality fails if $\|\cdot\|_{\frac{n}{n-\alpha / 2}}$ is replaced by $\|\cdot\|_{L_{N}}$ for a Young function $N$ with

$$
\limsup _{s \rightarrow 0} s^{\frac{n-\alpha / 2}{n}} N(s)=\infty .
$$

(2) For any $\alpha \in(0,2)$ and $p \in \mathbb{R}$, there exists a constant $C>0$ such that for all $f \in$ $L_{N_{\alpha}^{l o g, p,-}}\left(\mathbb{Z}^{n}\right)$

$$
\|f\|_{N_{\alpha}^{l o g, p,-}} \leq C \sum_{x, y \in \mathbb{Z}^{n}, x \neq y}|f(x)-f(y)| \frac{\{\log (2+|x-y|)\}^{p}}{|x-y|^{n+\alpha / 2}} .
$$

This inequality fails if $N_{\alpha}^{\text {log,p,- }}$ is replaced by a Young function $N$ with

$$
\limsup _{s \rightarrow 0} \frac{N(s)}{N_{\alpha}^{l o g, p,-}(s)}=\infty
$$

Proof. According to the proof of Theorem 1.1, it suffices to construct a symmetric subMarkov semigroup $P_{t}$ on $L^{2}(\mu)$ such that the associated Dirichlet form $(\mathscr{E}, \mathscr{D}(\mathscr{E}))$ is comparable with

$$
\mathscr{E}_{\alpha}(f, f):=\frac{1}{2} \sum_{x, y \in \mathbb{Z}^{n}, x \neq y} \frac{(f(x)-f(y))^{2}}{|x-y|^{n+\alpha}}, \quad \mathscr{D}\left(\mathscr{E}_{\alpha}\right):=\left\{f \in L^{2}(\mu), \mathscr{E}_{\alpha}(f, f)<\infty\right\},
$$

i.e., $\mathscr{D}(\mathscr{E})=\mathscr{D}\left(\mathscr{E}_{\alpha}\right)$ and there exists a constant $C \geq 1$ such that

$$
C^{-1} \mathscr{E}(f, f) \leq \mathscr{E}_{\alpha}(f, f) \leq C \mathscr{E}(f, f), \quad f \in \mathscr{D}(\mathscr{E})=\mathscr{D}\left(\mathscr{E}_{\alpha}\right)
$$

and moreover, both (3.1) and (3.2) are satisfied for $P_{t}$, where in (3.2)

$$
\left\|\nabla P_{t} f\right\|_{\infty}:=\sup _{x, y \in \mathbb{Z}^{n},|x-y|=1}\left|P_{t} f(x)-P_{t} f(y)\right| .
$$


Condition (3.1) can be easily deduced from the Nash inequality for $\left(\mathscr{E}_{\alpha}, \mathscr{D}\left(\mathscr{E}_{\alpha}\right)\right.$ ) (see for example [39, Proposition 2.1]), but to prove explicit gradient estimate (3.2) we need additional arguments. Below we first construct the required semigroup $P_{t}$ then verify these two estimates.

(1) Construction of $P_{t}$. Let $q_{k}(x, y)$ and $Q^{k}$ be the transition function and the semigroup for discrete time simple random walk $Y^{\prime}=\left(Y_{k}^{\prime}\right)_{k \geq 0}$ on $\mathbb{Z}^{n}$, respectively. It is known (see [48] or [29, Theorem 5.1]) that there are constants $c_{i}>0(i=1, \cdots 5)$ so that

$$
\begin{gathered}
q_{k}(x, y) \leq \frac{c_{1}}{k^{n / 2}} \exp \left(\frac{-c_{2}|x-y|^{2}}{k}\right), \quad x, y \in \mathbb{Z}^{n}, k \geq 1, \\
q_{k}(x, y)+q_{k+1}(x, y) \geq \frac{c_{3}}{k^{n / 2}} \exp \left(\frac{-c_{4}|x-y|^{2}}{k}\right), \quad x, y \in \mathbb{Z}^{n}, k \geq|x-y|
\end{gathered}
$$

and

$$
\begin{aligned}
\left\|\nabla Q^{k} f\right\|_{\infty} & :=\sup _{x \in \mathbb{Z}^{n}} \sup _{y \in \mathbb{Z}^{n},|y-x|=1}\left|Q^{k} f(y)-Q^{k} f(x)\right| \\
& \leq c_{5} k^{-1 / 2}\|f\|_{\infty}, \quad k \geq 1, f \in L^{\infty}\left(\mathbb{Z}^{n}\right) .
\end{aligned}
$$

Consider the discrete subordination of $Y^{\prime}$ by the Bernstein function $\psi(r)=r^{\alpha / 2}$ with $\alpha \in(0,2)$, see [6]. Denote by $X^{\prime}=\left(X_{k}^{\prime}\right)_{k \geq 0}$ the corresponding discrete time subordinated Markov chain on $\mathbb{Z}^{n}$, and by $p_{k}(x, y)$ the transition function of $X^{\prime}$. Then, according to $[6$, Proposition 2.3 and Example 2.1],

$$
p_{1}(x, y)=\sum_{k=1}^{\infty} c(\psi, k) q_{k}(x, y), \quad k \geq 1, x, y \in \mathbb{Z}^{n}
$$

where

$$
c(\psi, k)=\frac{\alpha}{2 \Gamma(1-\alpha / 2)} \frac{\Gamma(k-\alpha / 2)}{\Gamma(k+1)} .
$$

We claim that

$$
\frac{c_{0}^{-1}}{|x-y|^{n+\alpha}} \leq p_{1}(x, y) \leq \frac{c_{0}}{|x-y|^{n+\alpha}}, \quad x, y \in \mathbb{Z}^{n}, x \neq y
$$

holds for some constant $c_{0} \geq 1$. Indeed, by [25],

$$
c_{6}^{-1} k^{-(\alpha / 2+1)} \leq c(\psi, k) \leq c_{6} k^{-(\alpha / 2+1)}, \quad k \geq 1
$$

holds for some constant $c_{7} \geq 1$. Then, by (3.16), (3.18) and (3.20), we have

$$
p_{1}(x, y) \geq \frac{1}{2}\left(\sum_{k=1}^{\infty} c(\psi, k) q_{k}(x, y)+\sum_{k=1}^{\infty} c(\psi, k+1) q_{k+1}(x, y)\right)
$$




$$
\geq c_{7} \sum_{k=1}^{\infty} \frac{1}{k^{1+\alpha / 2}}\left(q_{k}(x, y)+q_{k+1}(x, y)\right) \geq c_{8} \sum_{k=|x-y|^{2}}^{\infty} \frac{1}{k^{1+(\alpha+n) / 2}} \geq \frac{c_{9}}{|x-y|^{n+\alpha}} .
$$

On the other hand, according to (3.15), (3.18) and (3.20),

$$
\begin{aligned}
p_{1}(x, y) & \leq c_{7} \sum_{k=1}^{\infty} \frac{1}{k^{1+\alpha / 2}} q_{k}(x, y) \\
& \leq c_{9}\left(\sum_{k=|x-y|^{2}}^{\infty} \frac{1}{k^{1+(\alpha+n) / 2}}+\sum_{k=1}^{|x-y|^{2}} \frac{1}{k^{1+(\alpha+n) / 2}} \exp \left(\frac{-c_{2}|x-y|^{2}}{k}\right)\right) \\
& \leq \frac{c_{10}}{|x-y|^{n+\alpha}} .
\end{aligned}
$$

Thus, (3.19) is proved.

Let $N_{t}$ be a Poisson process independent of $X^{\prime}$ and $Y^{\prime}$. Set $X_{t}=X_{N_{t}}^{\prime}$ and $Y_{t}=Y_{N_{t}}^{\prime}$ for all $t \geq 0$. Therefore, by (3.19), $X=\left(X_{t}\right)_{t \geq 0}$ is a continuous time symmetric Markov chain on $\mathbb{Z}^{n}$ such that the associated Dirichlet form $(\mathscr{E}, \mathscr{D}(\mathscr{E}))$ is comparable with $\mathscr{E}_{\alpha}$, i.e., (3.14) holds for some constant $C>1$. Let $P_{t}$ be the Markov semigroup of $X_{t}$.

(2) Proofs of (3.1) and (3.2). Let $Q_{t}$ be the Markov semigroup of $Y_{t}$. We have

$$
Q_{t}=\mathrm{e}^{-t} \sum_{k=0}^{\infty} \frac{t^{k} Q^{k}}{k !}, \quad t>0 .
$$

Then, by (3.15), for any $f \in L^{\infty}\left(\mathbb{Z}^{n}\right)$ and $t>0$,

$$
\begin{aligned}
\left\|Q_{t} f\right\|_{\infty} & \leq \mathrm{e}^{-t}\|f\|_{\infty}+c_{1} \mathrm{e}^{-t} \sum_{k=1}^{\infty} \frac{t^{k} k^{-n / 2}}{k !}\|f\|_{\infty} \\
& \leq \mathrm{e}^{-t}\|f\|_{\infty}+c_{11} t^{-d / 2}\|f\|_{\infty} \leq \frac{c_{12}}{t^{n / 2}}\|f\|_{\infty},
\end{aligned}
$$

where in the second inequality we have used the expansion for inverse moments of Poisson distribution, see [56, (29) in Corollary 3]. By (3.17), we also have that for any $f \in L^{\infty}\left(\mathbb{Z}^{n}\right)$ and $t>0$,

$$
\left\|\nabla Q_{t} f\right\|_{\infty} \leq \mathrm{e}^{-t}\|f\|_{\infty}+c_{6} \mathrm{e}^{-t} \sum_{k=1}^{\infty} \frac{t^{k} k^{-1 / 2}}{k !}\|f\|_{\infty} \leq \frac{c_{13}}{t^{1 / 2}}\|f\|_{\infty},
$$

where in the last inequality we have used again [56, (29) in Corollary 3].

On the other hand, let $S_{t}$ be the $\alpha / 2$-subordinator, which is independent of $X, X^{\prime}, Y$ and $Y^{\prime}$. According to [38, Proposition 1.2], we know that $X_{t}$ and $Y_{S_{t}}$ enjoy the same distribution. That is,

$$
P_{t} f=\int_{0}^{\infty} Q_{s} f \mathbb{P}\left(S_{t} \in \mathrm{d} s\right), \quad t \geq 0, f \in L^{\infty}\left(\mathbb{Z}^{n}\right) .
$$


This along with (3.21) and (3.22) yields

$$
\frac{\left\|P_{t} f\right\|_{\infty}}{\|f\|_{\infty}} \leq c_{12} \int_{0}^{\infty} s^{-n / 2} \mathbb{P}\left(S_{t} \in \mathrm{d} s\right) \leq c_{14} t^{-n / \alpha}, \quad t \geq 0, f \in L^{\infty}\left(\mathbb{Z}^{n}\right)
$$

and

$$
\frac{\left\|\nabla P_{t} f\right\|_{\infty}}{\|f\|_{\infty}} \leq c_{13} \int_{0}^{\infty} s^{-1 / 2} \mathbb{P}\left(S_{t} \in \mathrm{d} s\right) \leq c_{15} t^{-1 / \alpha}, \quad t \geq 0, f \in L^{\infty}\left(\mathbb{Z}^{n}\right),
$$

where we used the fact that $\mathbb{E} S_{t}^{-\lambda}=c_{\alpha, \lambda} t^{-2 \lambda / \alpha}$ for all $t, \lambda>0$, see [44, (25.5)]. Therefore, (3.1) and (3.2) hold.

\section{Isoperimetric inequalities for $\alpha$-stable-like Dirichlet forms: a perturbation argument}

Let $n \geq 2$ and $\alpha \in(0,2)$. Let $W \in \mathscr{B}\left(\mathbb{R}^{n}\right)$ be such that $\mu_{W}(\mathrm{~d} x):=\mathrm{e}^{-W(x)} \mathrm{d} x$ is a probability measure. Consider the following $\alpha$-stable-like Dirichlet form $\left(\mathscr{E}_{\alpha, W}, \mathscr{D}\left(\mathscr{E}_{\alpha, W}\right)\right)$ :

$$
\begin{aligned}
\mathscr{E}_{\alpha, W}(f, f) & :=\int_{\mathbb{R}^{n} \times \mathbb{R}^{n}} \frac{|f(x)-f(y)|^{2}}{|x-y|^{n+\alpha}} \mathrm{d} y \mu_{W}(\mathrm{~d} x), \\
\mathscr{D}\left(\mathscr{E}_{\alpha, W}\right) & :=\left\{f \in L^{2}\left(\mathbb{R}^{n}, \mu_{W}\right): \mathscr{D}\left(\mathscr{E}_{\alpha, W}\right)(f, f)<\infty\right\} .
\end{aligned}
$$

Obviously, $C_{c}^{\infty}\left(\mathbb{R}^{n}\right) \subset \mathscr{D}\left(\mathscr{E}_{\alpha, W}\right)$. See $[55,18]$ for explicit criteria of Poincaré-type (i.e., Poincaré, weak Poincaré and super Poincaré) inequalities for this Dirichlet form.

Since it is not clear how to verify the regularity property (e.g. gradient estimates) for the associated semigroup $P_{t}$, we could not apply Theorem 2.2 for non-negative symmetric function $\gamma(x, y)$ satisfying $\gamma(x, y) \rightarrow 0$ as $y \rightarrow x$. So, in this section, we will establish isoperimetric inequalities for $\left(\mathscr{E}_{\alpha, W}, \mathscr{D}\left(\mathscr{E}_{\alpha, W}\right)\right)$ by using a perturbation argument. The main result of this section is the following.

Theorem 4.1. Let $n \geq 2$ and $\alpha \in(0,2)$. Let $W \in \mathscr{B}\left(\mathbb{R}^{n}\right)$ be such that $\mu_{W}(\mathrm{~d} x)=\mathrm{e}^{-W(x)} \mathrm{d} x$ is a probability measure. Set

$$
\Phi(l):=\inf _{|x| \geq l} \frac{\mathrm{e}^{W(x)}}{|x|^{n+\alpha / 2}}, \quad l \geq 1 .
$$

(1) If $\lim _{l \rightarrow \infty} \Phi(l)>0$, then there are constants $c_{1}, c_{2}>0$ such that for any $f \in C_{c}^{\infty}\left(\mathbb{R}^{n}\right)$,

$$
\mu_{W}\left(f^{2}\right) \leq c_{1} \int_{\mathbb{R}^{n} \times \mathbb{R}^{n}} \frac{\left|f^{2}(x)-f^{2}(y)\right|}{|x-y|^{n+\alpha / 2}} \mathrm{~d} y \mu_{W}(\mathrm{~d} x)+c_{2} \mu_{W}(|f|)^{2} .
$$

(2) Let $W$ be locally Lipschitz continuous. If $\lim _{l \rightarrow \infty} \Phi(l)=\infty$, then for any $r>0$ and $f \in C_{c}^{\infty}\left(\mathbb{R}^{d}\right)$,

$$
\mu_{W}\left(f^{2}\right) \leq r \int_{\mathbb{R}^{n} \times \mathbb{R}^{n}} \frac{\left|f^{2}(x)-f^{2}(y)\right|}{|x-y|^{n+\alpha / 2}} \mathrm{~d} y \mu_{W}(\mathrm{~d} x)+\beta(r) \mu_{W}(|f|)^{2},
$$


where

$$
\begin{array}{r}
\beta(r)=\inf \left\{2 c_{1}\left(s^{-2 n / \alpha}+s^{-n}\right)\left(\sup _{|z| \leq l+1} \mathrm{e}^{W(z) / 2}\right): s+\frac{1}{\Phi(l-1)} \leq c_{2}(r \wedge 1)\right. \\
\left.\quad \text { and } \sup _{|z| \leq l+2} \mathrm{e}^{2|\nabla W(z)|} \leq \frac{c_{3}}{s}\right\}
\end{array}
$$

with some constants $c_{1}, c_{2}, c_{3}>0$.

To prove Theorem 4.1, we need the following Poincaré type isoperimetric inequality for the truncated $\alpha$-stable Dirichlet from on $\mathbb{R}^{n}$.

Lemma 4.2. There is a constant $c>0$ such that for all $f \in C_{c}^{\infty}\left(\mathbb{R}^{n}\right)$,

$$
\int f(x)^{2} \mathrm{~d} x \leq r \int_{\{|x-y| \leq 1\}} \frac{\left|f^{2}(x)-f^{2}(y)\right|}{|x-y|^{n+\alpha / 2}} \mathrm{~d} x \mathrm{~d} y+c\left(r^{-2 n / \alpha}+r^{-n}\right)\left(\int|f|(x) \mathrm{d} x\right)^{2}, \quad r>0 .
$$

Proof. This follows from (3.9) and Corollary 2.7(1).

For any $D \subset \mathbb{R}^{n}$, consider the isoperimetric constant

$$
\begin{aligned}
\kappa_{W}(D) & :=\frac{1}{2} \inf \left\{\frac{1}{\mu_{W}(A)} \int_{A \times A^{c}} \frac{\mathrm{e}^{W(x)}+\mathrm{e}^{W(y)}}{|x-y|^{n+\alpha / 2}} \mu_{W}(\mathrm{~d} x) \mu_{W}(\mathrm{~d} y): A \subset D, \mu_{W}(A)>0\right\} \\
& =\inf \left\{\frac{1}{\mu_{W}(f)} \int \frac{|f(x)-f(y)|}{|x-y|^{n+\alpha / 2}} \mathrm{~d} y \mu_{W}(\mathrm{~d} x): f \geq 0,\left.f\right|_{D^{c}}=0, \mu_{W}(f)>0\right\},
\end{aligned}
$$

where the second equality in (4.4) can be verified by the co-area formula, see [30, Theorem $3.1]$.

Lemma 4.3. Let $n \geq 2$ and $\alpha \in(0,2)$. Let $B_{l}=\{|\cdot|<l\}$ for $l>0$.

(1) If

$$
\lim _{l \rightarrow \infty} \kappa_{W}\left(B_{l}^{c}\right)>0,
$$

then (4.2) holds with some constants $c_{1}, c_{2}>0$.

(2) Let $W$ be locally Lipschitz continuous. If

$$
\lim _{l \rightarrow \infty} \kappa_{W}\left(B_{l}^{c}\right)=\infty
$$

then (4.3) holds with

$$
\begin{array}{r}
\beta(r)=\inf \left\{2 c_{1}\left(s^{-2 n / \alpha}+s^{-n}\right)\left(\sup _{|z| \leq l+1} \mathrm{e}^{W(z) / 2}\right): s+\frac{1}{\kappa_{W}\left(B_{l-1}^{c}\right)} \leq c_{2}(r \wedge 1)\right. \\
\left.\quad \text { and } \sup _{|z| \leq l+2} \mathrm{e}^{2|\nabla W(z)|} \leq \frac{c_{3}}{s}\right\}
\end{array}
$$

for some constants $c_{1}, c_{2}, c_{3}>0$. 
Proof. For any $l>k \geq 1$, let $\psi_{l, k} \in C_{c}^{1}\left(\mathbb{R}^{n}\right)$ such that $\psi_{l, k}(x)=1$ for all $|x| \leq l, \psi_{l, k}(x)=0$ for all $|x|>l+k$, and $\left|\nabla \psi_{l, k}\right| \leq 2 / k$ on $\mathbb{R}^{n}$. Then, according to Lemma 4.2, for any $f \in C_{c}^{\infty}\left(\mathbb{R}^{n}\right)$ and $r>0$,

$$
\begin{aligned}
& \int f^{2}(x) \mathrm{e}^{-W(x)} \mathbf{1}_{\{|x| \leq l\}} d x \\
& \leq \int f^{2}(x) \mathrm{e}^{-W(x)} \psi_{l, k}(x) \mathrm{d} x \\
& \leq r \int_{\{|x-y| \leq 1\}} \frac{\left|f^{2}(x) \mathrm{e}^{-W(x)} \psi_{l, k}(x)-f^{2}(y) \mathrm{e}^{-W(y)} \psi_{l, k}(y)\right|}{|x-y|^{n+\alpha / 2}} \mathrm{~d} x \mathrm{~d} y \\
& +c_{1}\left(r^{-2 n / \alpha}+r^{-n}\right)\left(\int|f|(x) \mathrm{e}^{-W(x) / 2} \psi_{l, k}(x)^{1 / 2} \mathrm{~d} x\right)^{2} \\
& \leq r \int_{\{|x-y| \leq 1\}} \frac{\left|f^{2}(x)-f^{2}(y)\right|}{|x-y|^{n+\alpha / 2}} \mathrm{~d} y \mathrm{e}^{-W(x)} \psi_{l, k}(x) \mathrm{d} x \\
& +r \int f^{2}(x) \mathrm{e}^{-W(x)} \mathrm{d} x \int_{\{|x-y| \leq 1\}} \frac{\left|\psi_{l, k}(x)-\psi_{l, k}(y)\right|}{|x-y|^{n+\alpha / 2}} \mathrm{~d} y \\
& +r \int f^{2}(x) \mathrm{e}^{-W(x)} \mathrm{d} x \int_{\{|x-y| \leq 1\}} \frac{\left|1-\mathrm{e}^{W(x)-W(y)}\right|}{|x-y|^{n+\alpha / 2}} \psi_{l, k}(y) \mathrm{d} y \\
& +c_{1}\left(r^{-2 n / \alpha}+r^{-n}\right)\left(\sup _{|z| \leq l+k} \mathrm{e}^{W(z) / 2}\right)\left(\int|f|(x) \mathrm{e}^{-W(x)} \mathrm{d} x\right)^{2} \\
& \leq r \int_{\{|x-y| \leq 1\}} \frac{\left|f^{2}(x)-f^{2}(y)\right|}{|x-y|^{n+\alpha / 2}} \mathrm{~d} y \mathrm{e}^{-W(x)} \mathrm{d} x \\
& +\frac{c_{2} r}{k}\left(\int f^{2}(x) \mathrm{e}^{-W(x)} \mathrm{d} x\right)+c_{3} r\left(\sup _{|z| \leq l+k+1} \mathrm{e}^{2|\nabla W(z)|}\right)\left(\int f^{2}(x) \mathrm{e}^{-W(x)} \mathrm{d} x\right) \\
& +c_{1}\left(r^{-2 n / \alpha}+r^{-n}\right)\left(\sup _{|z| \leq l+k} \mathrm{e}^{W(z) / 2}\right)\left(\int|f|(x) \mathrm{e}^{-W(x)} \mathrm{d} x\right)^{2},
\end{aligned}
$$

where in the last inequality we have used the facts that for all $x \in \mathbb{R}^{d}$,

$$
\int_{\{|x-y| \leq 1\}} \frac{\left|\psi_{l, k}(x)-\psi_{l, k}(y)\right|}{|x-y|^{n+\alpha / 2}} \mathrm{~d} y \leq\left(\sup _{z \in \mathbb{R}^{n}}\left|\nabla \psi_{l, k}\right|(z)\right) \int_{\{|x-y| \leq 1\}} \frac{1}{|x-y|^{n-1+\alpha / 2}} \mathrm{~d} y \leq \frac{c_{2}}{k}
$$

and, by the elementary inequality $\left|\mathrm{e}^{r}-1\right| \leq \mathrm{e}^{|r|}|r| \leq \mathrm{e}^{2|r|}$ for all $r \in \mathbb{R}$,

$$
\begin{aligned}
& \int_{\{|x-y| \leq 1\}} \frac{\left|1-\mathrm{e}^{W(x)-W(y)}\right|}{|x-y|^{n+\alpha / 2}} \psi_{l, k}(y) \mathrm{d} y \\
& \leq \sup _{|x| \leq l+k+1} \int_{\{|x-y| \leq 1,|y| \leq l+k\}} \frac{\left|1-\mathrm{e}^{W(x)-W(y)}\right|}{|x-y|^{n+\alpha / 2}} \mathrm{~d} y \\
& \leq \sup _{|x| \leq l+k+1} \int_{\{|x-y| \leq 1,|y| \leq l+k\}} \frac{\mathrm{e}^{|W(x)-W(y)|}|W(x)-W(y)|}{|x-y|^{n+\alpha / 2}} \mathrm{~d} y
\end{aligned}
$$




$$
\begin{aligned}
& \leq\left(\sup _{|z| \leq l+k+1} \mathrm{e}^{|\nabla W(z)|}|\nabla W(z)|\right) \int_{\{|x-y| \leq 1\}} \frac{1}{|x-y|^{n-1+\alpha / 2}} \mathrm{~d} y \\
& \leq c_{3}\left(\sup _{|z| \leq l+k+1} \mathrm{e}^{2|\nabla W(z)|}\right) .
\end{aligned}
$$

On the other hand, by the definition of $\kappa_{W}\left(B_{l-k}^{c}\right)$, we have

$$
\begin{aligned}
\int & f^{2}(x) \mathrm{e}^{-W(x)}\left(1-\mathbf{1}_{\{|x| \leq l\}}\right) d x \\
\leq & \int f^{2}(x) \mathrm{e}^{-W(x)}\left(1-\psi_{l-k, k}(x)\right) \mathrm{d} x \\
\leq & \frac{1}{\kappa_{W}\left(B_{l-k}^{c}\right)} \int \frac{\left|f^{2}(x)\left(1-\psi_{l-k, k}(x)\right)-f^{2}(y)\left(1-\psi_{l-k, k}(y)\right)\right|}{|x-y|^{n+\alpha / 2}} \mathrm{~d} y \mathrm{e}^{-W(x)} \mathrm{d} x \\
\leq & \frac{1}{\kappa_{W}\left(B_{l-k}^{c}\right)} \int \frac{\left|f^{2}(x)-f^{2}(y)\right|}{|x-y|^{n+\alpha / 2}} \mathrm{~d} y \mathrm{e}^{-W(x)} \mathrm{d} x \\
& +\frac{1}{\kappa_{W}\left(B_{l-k}^{c}\right)} \int \frac{\left|f^{2}(x) \psi_{l-k, k}(x)-f^{2}(y) \psi_{l-k, k}(y)\right|}{|x-y|^{n+\alpha / 2}} \mathrm{~d} y \mathrm{e}^{-W(x)} \mathrm{d} x \\
\leq & \frac{2}{\kappa_{W}\left(B_{l-k}^{c}\right)} \int \frac{\left|f^{2}(x)-f^{2}(y)\right|}{|x-y|^{n+\alpha / 2}} \mathrm{~d} y \mathrm{e}^{-W(x)} \mathrm{d} x \\
& +\frac{1}{\kappa_{W}\left(B_{l-k}^{c}\right)} \int f^{2}(x) \mathrm{e}^{-W(x)} \mathrm{d} x \int \frac{\left|\psi_{l-k, k}(x)-\psi_{l-k, k}(y)\right|}{|x-y|^{n+\alpha / 2}} \mathrm{~d} y \\
\leq & \frac{2}{\kappa_{W}\left(B_{l-k}^{c}\right)} \int \frac{\left|f^{2}(x)-f^{2}(y)\right|}{|x-y|^{n+\alpha / 2}} \mathrm{~d} y \mathrm{e}^{-W(x)} \mathrm{d} x \\
& +\frac{c_{2}}{k \kappa_{W}\left(B_{l-k}^{c}\right)} \int f^{2}(x) \mathrm{e}^{-W(x)} \mathrm{d} x,
\end{aligned}
$$

where the last inequality follows from (4.7) again.

Combining both inequalities above, we have

$$
\begin{aligned}
\mu_{W}\left(f^{2}\right) \leq & \left(r+\frac{2}{\kappa_{W}\left(B_{l-k}^{c}\right)}\right) \int_{\mathbb{R}^{n} \times \mathbb{R}^{n}} \frac{\left|f^{2}(x)-f^{2}(y)\right|}{|x-y|^{n+\alpha / 2}} \mathrm{~d} y \mathrm{e}^{-W(x)} \mathrm{d} x \\
& +c_{1}\left(r^{-2 n / \alpha}+r^{-n}\right)\left(\sup _{|z| \leq l+k} \mathrm{e}^{W(z) / 2}\right) \mu_{W}(|f|)^{2} \\
& +\left(\left(\frac{c_{2}}{k}+c_{3} \sup _{|z| \leq l+k+1} \mathrm{e}^{2|\nabla W(z)|}\right) r+\frac{c_{2}}{k \kappa_{W}\left(B_{l-k}^{c}\right)}\right) \mu_{W}\left(f^{2}\right) .
\end{aligned}
$$

(1) Taking $l=2 k$, we have

$$
\begin{aligned}
& \left(\frac{c_{2}}{k}+c_{3} \sup _{|z| \leq l+k+1} \mathrm{e}^{2|\nabla W(z)|}\right) r+\frac{c_{2}}{k \kappa_{W}\left(B_{l-k}^{c}\right)} \\
& =\left(\frac{c_{2}}{k}+c_{3} \sup _{|z| \leq 3 k+1} \mathrm{e}^{2|\nabla W(z)|}\right) r+\frac{c_{2}}{k \kappa_{W}\left(B_{k}^{c}\right)} .
\end{aligned}
$$


Since $\kappa_{W}\left(B_{l}^{c}\right)$ is increasing with respect to $l$, under (4.5) we can choose $k \geq 1$ large enough and then take $r>0$ small enough such that

$$
\left(\frac{c_{2}}{k}+c_{3} \sup _{|z| \leq 3 k+1} \mathrm{e}^{2|\nabla W(z)|}\right) r+\frac{c_{2}}{k \kappa_{W}\left(B_{k}^{c}\right)} \leq \frac{1}{2}
$$

This along with (4.8) yields (4.2).

(2) Taking $k=1$ in (4.8) and using (4.6), we know that (4.3) holds with

$$
\begin{gathered}
\beta(r)=\inf \left\{2 c_{1}\left(s^{-2 n / \alpha}+s^{-n}\right)\left(\sup _{|z| \leq l+1} \mathrm{e}^{W(z) / 2}\right): s+\frac{2}{\kappa_{W}\left(B_{l-1}^{c}\right)} \leq \frac{r}{2}\right. \\
\text { and } \left.\left(c_{2}+c_{3} \sup _{|z| \leq l+2} \mathrm{e}^{2|\nabla W(z)|}\right) s+\frac{c_{2}}{\kappa_{W}\left(B_{l-1}^{c}\right)} \leq \frac{1}{2}\right\} .
\end{gathered}
$$

Note that

$$
\begin{array}{r}
\beta(r) \leq \inf \left\{2 c_{1}\left(s^{-2 n / \alpha}+s^{-n}\right)\left(\sup _{|z| \leq l+1} \mathrm{e}^{W(z) / 2}\right): s+\frac{1}{\kappa_{W}\left(B_{l-1}^{c}\right)} \leq c_{4}(r \wedge 1)\right. \\
\left.\quad \text { and } \sup _{|z| \leq l+2} \mathrm{e}^{2|\nabla W(z)|} s \leq c_{5}\right\} .
\end{array}
$$

Then, we prove (4.3) with the desired $\beta$.

Lemma 4.4. For any $\alpha \in(0,2)$, there is a constant $c_{0}>0$ such that

$$
\kappa_{W}\left(B_{l}^{c}\right) \geq c_{0} \inf _{|x| \geq l} \frac{\mathrm{e}^{W(x)}}{|x|^{n+\alpha / 2}}, \quad l \geq 1 .
$$

Proof. According to the definition of $\kappa_{W}\left(B_{l}^{c}\right)$, we have

$$
\begin{aligned}
\kappa_{W}\left(B_{l}^{c}\right) & \geq \frac{1}{2} \inf _{A \subset B_{l}^{c}} \inf _{x \in A} \int_{A^{c}} \frac{\mathrm{e}^{W(x)-W(y)}+1}{|x-y|^{n+\alpha / 2}} \mathrm{~d} y \geq \frac{1}{2} \inf _{|x| \geq l} \int_{\{|y|<l\}} \frac{\mathrm{e}^{W(x)-W(y)}+1}{|x-y|^{n+\alpha / 2}} \mathrm{~d} y \\
& \geq c_{1} \inf _{|x| \geq l} \mathrm{e}^{W(x)} \int_{\{|y| \leq 1\}} \frac{1}{|x-y|^{n+\alpha / 2}} \mathrm{~d} y \geq c_{2} \inf _{|x| \geq l} \frac{\mathrm{e}^{W(x)}}{|x|^{n+\alpha / 2}} .
\end{aligned}
$$

This proves the desired assertion.

Theorem 4.1 is a direct consequence of Lemmas 4.3 and 4.4 , and so we omit the proof here.

The example below indicates that Theorem 4.1 is sharp in some sense.

Example 4.5. Let $W(x)=\frac{1}{2}(n+\varepsilon) \log \left(1+|x|^{2}\right)+c_{n, \varepsilon}$ for $\varepsilon>0$.

(1) (4.2) holds if and only if $\varepsilon \geq \alpha / 2$. 
(2) (4.3) holds if and only if $\varepsilon>\alpha / 2$. Furthermore, when $\varepsilon>\alpha / 2$, (4.3) holds with

$$
\beta(r)=c_{1}(1 \wedge r)^{-\frac{2 n}{\alpha}-\frac{n+\varepsilon}{2 \varepsilon-\alpha}} .
$$

Proof. The sufficiency for both conclusions is easily seen from Theorem 4.1. To verify the necessary, we will make use of the reference functions used in [55, Corollary 1.1]. For any $l \geq 1$, define $g_{l} \in C^{\infty}\left(\mathbb{R}^{n}\right)$ such that $\left|\nabla g_{l}\right| \leq 2 / l$ and

$$
g_{l}(x) \begin{cases}=0, & \text { if }|x| \leq l, \\ \in[0,1], & \text { if }|x| \in[l, 2 l], \\ =1, & \text { if }|x| \geq 2 l .\end{cases}
$$

Then there exists a constant $c_{0}>0$ independent of $l$ such that for all $x \in \mathbb{R}^{n}$ and $l \geq 1$,

$$
\begin{aligned}
\int_{\mathbb{R}^{n}} \frac{\left|g_{l}^{2}(y)-g_{l}^{2}(x)\right|}{|x-y|^{n+\alpha / 2}} \mathrm{~d} y & \leq 2 \int_{\mathbb{R}^{n}} \frac{\left|g_{l}(y)-g_{l}(x)\right|}{|x-y|^{n+\alpha / 2}} \mathrm{~d} y \\
& \leq \frac{4}{l} \int_{\{|x-y| \leq l\}} \frac{1}{|y-x|^{n+\alpha / 2-1}} \mathrm{~d} y+2 \int_{\{|x-y| \geq l\}} \frac{1}{|x-y|^{n+\alpha / 2}} \mathrm{~d} y \\
& \leq \frac{c_{0}}{l^{\alpha / 2}}
\end{aligned}
$$

Obviously,

$$
\mu_{W}\left(g_{l}^{2}\right) \geq \frac{c_{1}}{l^{\varepsilon}}, \quad \mu_{W}\left(g_{l}\right)^{2} \leq \frac{c_{2}}{l^{2 \varepsilon}}, \quad l \geq 1
$$

hold for some constants $c_{1}, c_{2}>0$. Note that, since $1-g_{l} \in C_{c}^{\infty}\left(\mathbb{R}^{n}\right)$, we can directly apply $g_{l}$ into (4.2) and (4.3).

(1) Combining (4.9) with (4.10), we see that for any $c>0$,

$$
\lim _{l \rightarrow \infty} \frac{1}{\mu_{W}\left(g_{l}^{2}\right)-c \mu_{W}\left(g_{l}\right)^{2}} \int \frac{\left|g_{l}^{2}(y)-g_{l}^{2}(x)\right|}{|x-y|^{n+\alpha / 2}} \mathrm{~d} y \mu_{W}(\mathrm{~d} x) \leq \lim _{l \rightarrow \infty} \frac{c_{0} l^{-\alpha / 2}}{c_{1} l^{-\varepsilon}-c c_{2} l^{-2 \varepsilon}}=0
$$

provided $\varepsilon \in(0, \alpha / 2)$. Thus, the inequality (4.2) does not hold.

(2) We first prove that if $\varepsilon \leq \alpha / 2$, then for any $\beta:(0, \infty) \rightarrow(0, \infty)$ the inequality (4.3) does not hold. Indeed, if this inequality holds, then, by (4.9) and (4.10),

$$
\frac{c_{1}}{l^{\varepsilon}} \leq \frac{c_{0} r}{l^{\alpha / 2}}+\frac{c_{2} \beta(r)}{l^{2 \varepsilon}}, \quad r>0, l \geq 1
$$

holds for some constants $c_{0}, c_{1}, c_{2}>0$. Since $\varepsilon \in(0, \alpha / 2]$, we obtain

$$
c_{1} \leq \lim _{l \rightarrow \infty} \frac{c_{0} r}{l^{(\alpha / 2)-\varepsilon}}+\lim _{l \rightarrow \infty} \frac{c_{2} \beta(r)}{l^{\varepsilon}} \leq c_{0} r, \quad r>0 .
$$

Letting $r \rightarrow 0$ we conclude that $c_{1} \leq 0$, which is however impossible. Furthermore, by Theorem 4.1(2), it is easy to prove (4.3) with the desired rate function $\beta(r)$. 
We close this section with the following remark.

Remark 4.6. Isoperimetric inequalities for local Dirichlet forms with finite reference measures can not be obtained by simply letting $\alpha \rightarrow 2-$ in the corresponding inequalities given in Theorem 4.1. Actually, it is known that Cheeger-type (dimension free) isoperimetric inequalities for local Dirichlet forms with respect to the probability measure $\mu_{\theta}=Z_{\theta}^{-1} e^{-|x|^{\theta}}$ holds if and only if $\theta \geq 1$, see [47, 9,3]. However, according to Example 4.5(1), isoperimetric inequalities for the local Dirichlet form $\left(\mathscr{E}_{\alpha, W}, \mathscr{D}\left(\mathscr{E}_{\alpha, W}\right)\right)$ given by (4.1) holds for the probability measure $\mu_{\theta}(d x)=Z_{\theta}^{-1}(1+|x|)^{-d-\theta}$ with $\theta \geq \alpha / 2$. So, the properties of isoperimetric inequalities for local Dirichlet forms with finite reference measures and these for nonlocal Dirichlet forms are essentially different. The same for Poincaré-type inequalities for non-local Dirichlet forms studied in [55, 18].

Acknowledgement. Supported in part by NNSFC (11431014,11522106,11626245,11626250, 11831014), the Program for Probability and Statistics: Theory and Application (No. IRTL1704) and the Program for Innovative Research Team in Science and Technology in Fujian Province University (IRTSTFJ). The authors would like to thank Professor Takashi Kumagai and the referee for their helpful comments.

\section{References}

[1] R.A. Adams, Sobolev Spaces, Academic Press, New York, 1975.

[2] L. Ambrosio, G. De Philippis, L. Martinazzi, Gamma-convergence of nonlocal perimeter functionals, Manuscripta Math. 134(2011), 377-403.

[3] F. Barthe, P. Cattiaux, C. Roberto, Interpolated inequalities between exponential and Gaussian, Orlicz hypercontractivity and application to isoperimetry, Revista Mat. Iberoamericana, $22(2006), 993-1066$.

[4] F. Barthe, P. Cattiaux, C. Roberto, Isoperimetry between exponential and Gaussian, Electronic J. Proba. 12(2007), 1212-1237.

[5] D. Bakry, T. Coulhon, M. Ledoux, and L. Saloff-Coste, Sobolev inequalities in disguise, Indiana Univ. Math. J., 44(1995), 1033-1074.

[6] A. Bendikov, L. Saloff-Coste, Random walks on groups and discrete subordination, Math. Nachr. 285(2012), 580-605.

[7] O.V. Besov, On a certain family of functional spaces. Imbedding and continuation theorems, Dokl. Akad. Nauk SSSR 126(1959), 1163-1165.

[8] O.V. Besov, On some conditions of membership in $L_{p}$ for derivatives of periodic functions, Naučn. Dokl. Vyss. Skoly. Fiz.-Mat. Nauki (1959), 13-17.

[9] S.G. Bobkov, C. Houdré, Isoperimetric constants for product probability measures, Ann. Probab. 25(1997), 184-205. 
[10] J. Bourgain, H. Brezis, P. Mironescu, Limiting embedding theorems for $W_{s, p}$ when $s \uparrow 1$ and applications, J. Anal. Math. 87(2002), 77-101,

[11] P. Buser, A note on the isoperimetric constant, Ann. Sci. École Norm. Sup. 15(1992), 213230.

[12] L. Caffarelli, J.M. Roquejoffre, O. Savin, Non-local minimal surfaces, Comm. Pure Appl. Math. 63(2010), 1111-1144.

[13] L. Caffarelli, E. Valdinoci, Uniform estimates and limiting arguments for nonlocal minimal surfaces, Calc. Var. Partial Differential Equations 41(2011), 203-240.

[14] J. Cheeger, A lower bound for the smallest eigenvalue of the Laplacian, in: Problem in Analysis, a Symposium in Honor of S. Bochner, Princeton Uinv. Press, Princeton, NJ, 1970, pp. 195-199.

[15] M.-F. Chen, Nash inequalities for general symmetric forms, Acta Mathematica Sinica, English Series 15(1999), 353-370.

[16] M.-F. Chen, Logarithmic Sobolev inequality for symmetric forms, Sci. in China(A) 43(2000), 601-608.

[17] M.-F. Chen, F.-Y. Wang, Cheeger's inequalities for general symmetric forms and existence criteria for spectral gap, Ann. Probab. 28(2000), 235-257.

[18] X. Chen, F.-Y. Wang, J. Wang, Functional inequalities for pure-jump Dirichlet forms, in: Festschrift Masatoshi Fukushima (Z-Q. Chen, N. Jacob, M. Takeda, T. Uemura Eds), World Scientific, New Jersey 2015, pp. 143-162.

[19] Z.-Q. Chen, P. Kim, T. Kumagai, Weighted Poincaré inequality and heat kernel estimates for finite range jump processes, Math. Ann. 342(2008), 833-883.

[20] Z.-Q. Chen, T. Kumagai, Heat kernel estimates for stable-like processes on $d$-sets, Stochastic Process Appl. 108(2003), 27-62.

[21] T. Coulhon, Heat kernel and isoperimetry on non-compact Riemannian manifolds, in: Contemporary Mathematics, vol. 338 (American Mathematical Society, Providence, 2003), pp. 65-99.

[22] E. B. Davies, Heat Kernels and Spectral Theory, Cambridge Univ. Press, Cambridge, 1989.

[23] J. Dávila, On an open question about functions of bounded variation, Calc. Var. Partial Differential Equations 15(2002), 517-529.

[24] S. Dipierro, A. Figalli, G. Palatucci, E. Valdinoci, Asymptotics of the s-perimeter as $s \searrow 0$, Discrete Contin. Dyn. Syst. 33(2013), 2777-2790.

[25] A. Erdélyi, F. G. Tricomi, The asymptotic expansion of a ratio of gamma functions, Pacific J. Math. 1(1951), 133-142. 
[26] A. Figalli, N. Fusco, F. Maggi, V. Millot, M. Morini, Isoperimetry and stability properties of balls with respect to nonlocal energies, Commun. Math. Phys. 336(2015), 441-507.

[27] R.L. Frank, R. Seiringer, Non-linear ground state representations and sharp Hardy inequalities, J. Funct. Anal. 255(2008), 3407-3430.

[28] N. Fusco, V. Millot, M. Morini, A quantitative isoperimetric inequality for fractional perimeters, J. Funct. Anal. 261(2011), 697-715.

[29] W. Hebisch, L. Saloff-Coste, Gaussian estimates for Markov chains and random walks on groups, Ann. Probab. 21(1993), 673-709.

[30] G.F. Lawler, A.D. Sokal, Bounds on the $L^{2}$ spectrum for Markov chain and Markov processes: a generalization of Cheeger's inequality, Trans. Amer. Math. Soc. 309(1998), 557-580.

[31] M. Ledoux, A simple proof of an inequality by P. Buser, Proc. Amer. Math. Soc. 121(1994), 951-958.

[32] Y.H. Mao, General Sobolev type inequalities for symmetric forms, J. Math. Anal. Appl. 338(2008), 1092-1099.

[33] Y.H. Mao, $L^{p}$-Poincaré inequality for general symmetric forms, Acta Mathematica Sinica, English Series 25(2009), 2055-2064.

[34] V. G. Maz'ja, Sobolev spaces, Springer Series in Soviet Mathematics, Springer, Berlin, 1985.

[35] V. Maz'ya, Lectures on isoperimetric and isocapacitary inequalities in the theory of Sobolev spaces, in: Contemporary Mathematics, vol. 338 (American Mathematical Society, Providence, 2003), pp. 307-340.

[36] V. Maz'ya, T. Shaposhnikova, On the Bourgain, Brezis, and Mironesce theorem concerning limiting embeddings of fractional Sobolev spaces, J. Funct. Anal. 195(2002), 230-238; Erratum, J. Funct. Anal. 201(2003), 298-300.

[37] E. Milman, On the role of convexity in functional and isoperimetric inequalities, Proc. London Math. Soc 99(2009), 32-66.

[38] A. Mimica, On subordinate random walks, Forum Mathematicum 29(2017), 653-664.

[39] M. Murugan, L. Saloff-Coste, Transition probability estimates for long range random walks, New York Journal of Mathematics 21(2015), 723-757.

[40] E. Di Nezza, G. Palatucci, E. Valdinoci, Hitchhiker's guide to the fractional Sobolev spaces, Bull. Sci. Math. 136(2012), 521-573.

[41] M.M. Rao, Z. D. Ren, Applications of Orlicz Spaces, Marcel Dekker, New York, 2002.

[42] M. Röckner, F.-Y. Wang, Weak Poincaré inequalities and $L^{2}$-convergence rates of Markov semigroups, J. Funct. Anal. 185(2001), 564-603. 
[43] L. Saloff-Coste, Lectures on finite Markov chains, in: Ecole d'Eté de Probabilités de SaintFlour XXVI-1996. Lecture Notes in Mathematics, vol. 1665 (Springer, Berlin, 1997), pp. 301-413.

[44] K. Sato, Lévy Processes and Infinitely Divisible Distributions, Cambridge University Press, Cambridge, 1999.

[45] R.L. Schilling, P. Sztonyk, J. Wang, Coupling property and gradient estimates of Lévy processes via the symbol, Bernoulli 18(2012), 1128-1149.

[46] L. Tartar, An Introduction to Sobolev Spaces and Interpolation Spaces, Lect. Notes Unione Mat. Ital., vol. 3, Springer-Verlag, Berlin, Heidelberg, 2007.

[47] M. Talagrand, A new isoperimetric inequality and the concentration of measure phenomenon, Israel Seminar (GAFA). Lecture Notes in Math. 1469, 94-124. Springer, Berlin, 1991.

[48] A. Telcs, The art of random walks, Lecture Notes in Mathematics, vol. 1855, Springer, Berlin, 2006.

[49] A. Visintin, Nonconvex functionals related to multiphase systems, SIAM J. Math. Anal. 21(1990), 1281-1304.

[50] F. Wang, Y.-H. Zhang, F-Sobolev inequality for general symmetric forms, Northeast. Math. J. 19(2003), 133-138.

[51] F.-Y. Wang, Functional inequalities for empty essential spectrum, J. Funct. Anal. 170(2000), 219-245.

[52] F.-Y. Wang, Functional inequalities, semigroup properties and spectrum estimates, Infin. Dimens. Anal. Quant. Probab. Relat. Topics 3(2000), 263-295.

[53] F.-Y. Wang, Sobolev type inequalities for general symmetric forms, Proc. Amer. Math. Soc 128(2000), 3675-3682.

[54] F.-Y. Wang, Functional Inequalities, Markov Processes and Spectral Theory, Science Press, Beijing, 2005.

[55] F.-Y. Wang, J. Wang, Functional inequalities for stable-like Dirichlet forms, J. Theor. Probab. 28(2015), 423-448.

[56] M. Žnidarič, Asymptotic expansion for inverse moments of Binomial and Poisson distributions, The Open Statistics \& Probability Journal 1(2009), 7-10. 